\title{
Vascular mineralocorticoid receptor regulates microRNA-155 to promote vasoconstriction and rising blood pressure with aging
}

Jennifer J. DuPont, ${ }^{1}$ Amy McCurley, ${ }^{1}$ Ana P. Davel, ${ }^{1,2}$ Joseph McCarthy, ${ }^{1}$ Shawn B. Bender, ${ }^{3,4,5}$ Kwangseok Hong, ${ }^{3,6}$ Yan Yang, ${ }^{3}$ Jeung-Ki Yoo, ${ }^{7}$ Mark Aronovitz, ${ }^{1}$ Wendy E. Baur, Demetra D. Christou, ${ }^{7}$ Michael A. Hill, ${ }^{3,6}$ and Iris Z. Jaffe ${ }^{1}$

${ }^{1}$ Molecular Cardiology Research Institute, Tufts Medical Center, Boston, Massachusetts, USA. ${ }^{2}$ Department of Structural and Functional Biology, Institute of Biology, University of Campinas-UNICAMP, São Paulo, Brazil. ${ }^{3}$ Dalton Cardiovascular Research Center, University of Missouri, Columbia, Missouri, USA. 'Research Service, Harry S. Truman Memorial Veterans' Hospital, Columbia, Missouri, USA. ${ }^{5}$ Department of Biomedical Sciences, University of Missouri, Columbia, Missouri, USA. ${ }^{6}$ Department of Medical Pharmacology and Physiology, University of Missouri School of Medicine, Columbia, Missouri, USA. 'Department of Applied Physiology and Kinesiology, University of Florida, Gainesville, Florida, USA.

Hypertension is nearly universal yet poorly controlled in the elderly despite proven benefits of intensive treatment. Mice lacking mineralocorticoid receptors in smooth muscle cells (SMC-MR$\mathrm{KO}$ ) are protected from rising blood pressure (BP) with aging, despite normal renal function. Vasoconstriction is attenuated in aged SMC-MR-KO mice, thus they were used to explore vascular mechanisms that may contribute to hypertension with aging. MicroRNA (miR) profiling identified miR-155 as the most down-regulated miR with vascular aging in MR-intact but not SMC-MR-KO mice. The aging-associated decrease in miR-155 in mesenteric resistance vessels was associated with increased mRNA abundance of MR and of predicted miR-155 targets Cav1.2 (L-type calcium channel (LTCC) subunit) and angiotensin type-1 receptor (AgtR1). SMC-MR-KO mice lacked these aging-associated vascular gene expression changes. In HEK293 cells, MR repressed miR-155 promoter activity. In cultured SMCs, miR-155 decreased Cav1.2 and AgtR1 mRNA. Compared to MR-intact littermates, aged SMC-MR-KO mice had decreased systolic BP, myogenic tone, SMC LTCC current, mesenteric vessel calcium influx, LTCC-induced vasoconstriction and angiotensin II-induced vasoconstriction and oxidative stress. Restoration of miR-155 specifically in SMCs of aged MRintact mice decreased Cav1.2 and AgtR1 mRNA and attenuated LTCC-mediated and angiotensin IIinduced vasoconstriction and oxidative stress. Finally, in a trial of MR blockade in elderly humans, changes in serum miR-155 predicted the BP treatment response. Thus, SMC-MR regulation of miR-155, Cav1.2 and AgtR1 impacts vasoconstriction with aging. This novel mechanism identifies potential new treatment strategies and biomarkers to improve and individualize antihypertensive therapy in the elderly.

Conflict of interest: The authors have declared that no conflict of interest exists.

Submitted: June 3, 2016 Accepted: August 2, 2016 Published: September 8, 2016

Reference information: JCI Insight. 2016;1(14):e88942. doi:10.1172/jci.insight.88942.

\section{Introduction}

Advancing age is a universal, potent, and currently un-modifiable risk factor for the development of hypertension and cardiovascular disease (1). Essential hypertension (high blood pressure (BP) without a secondary cause) is nearly an absolute consequence of aging in developed nations, affecting $60 \%$ of Americans over the age of 60 and $80 \%$ of the rapidly growing population over $80(2,3)$. Hypertension (HTN) is a substantial source of morbidity and mortality in the elderly, as high BP increases the risk of heart attack, stroke, vascular dementia, heart failure, kidney failure, and death (4). Despite this, only half of hypertensives over 50 years of age are controlled with current therapies $(4,5)$. Attempts to balance the benefits of BP lowering with the risks from adverse medication side effects resulted in the controversial 2014 recommendation by the Joint National Committee (JNC8) to target a more permissive BP goal of $150 / 90 \mathrm{mmHg}$ in those over the age of 60 (6). However, the recent results of the Systolic Blood Pressure Intervention (SPRINT) Trial have heightened the urgency 
for better BP control in older adults. SPRINT was stopped early due to a $27 \%$ reduction in mortality in older hypertensive patients randomized to a systolic BP target of $120 \mathrm{mmHg}$ compared to $140 \mathrm{mmHg}$ (7). Moreover, the recently published pre-specified SPRINT sub-study revealed even greater benefits from intensive BP control in people over age 75 , with a $34 \%$ reduction in major cardiovascular events and a $33 \%$ reduction in all-cause mortality without an increase in major adverse side effects (8). Thus, there is an urgent need to understand the molecular mechanisms contributing to the age-dependent rise in BP in order to identify treatment strategies to safely improve BP control with aging.

The kidney is an established target of many antihypertensive therapies because it is a critical regulator of BP by modulating sodium and water balance. Indeed, renal function declines with aging and combined with high sodium intake in the typical Western diet, contributes to volume retention and hypertension (9). Perhaps less appreciated is the concept that in response to increases in blood volume from renal mechanisms and vasoconstrictor pathways that are enhanced with aging, smooth muscle cells (SMC) in the resistance vasculature constrict, thereby increasing peripheral vascular resistance and exacerbating hypertension (10). Thus, the vasculature is also an important contributor to the development of hypertension and to BP control. In humans and rodents, vascular aging is associated with enhanced vascular oxidative stress and increased responsiveness to the vasoconstrictor hormone angiotensin II (AngII) and these factors contribute to enhanced vasoconstriction with aging (11). However, the molecular mechanisms driving these vascular changes that contribute to hypertension with aging have not been elucidated.

Although the adrenal hormone aldosterone and its mineralocorticoid receptor (MR) are well known regulators of $\mathrm{BP}$ by promoting renal sodium reabsorption in the kidney, we previously demonstrated that MR is also expressed and functional in human vascular SMC (12-14). Moreover, we found that mice with MR specifically deleted from SMC in adulthood (SMC-MR-KO mice), are protected from the modest aging-associated rise in systolic BP that occurs in MR-intact mice, despite no change in renal function, sodium handling, or serum aldosterone levels (15). Rather, aged SMC-MR-KO mice had decreased vasoconstriction in response to increased intravascular pressure (termed myogenic tone) and were protected from AngII-induced vasoconstriction and vascular oxidative stress (15), important drivers of vascular dysfunction and hypertension with aging. Thus, the SMC-MR-KO mouse was used to explore mechanisms driving vasoconstriction with aging as these mechanisms may contribute to hypertension in elderly humans and could suggest new therapeutic strategies to improve BP control. We discovered that with aging, MR expression rises in resistance vessels along with a decline in microRNA (miR)-155 and increased expression of predicted miR-155 targets including the L-type calcium channel (LTCC) subunit Cav1.2 and the angiotensin type-1 receptor (AgtR1), genes that contribute to vasoconstriction and oxidative stress in aging mice. Restoration of miR-155 in aged vessels decreased target gene expression and vasoconstriction. Finally, in older humans, changes in miR-155 levels in response to MR antagonism correlated with improved BP response to therapy.

\section{Results}

Smooth muscle cell mineralocorticoid receptor modulates vascular microRNA expression changes with aging. MicroRNAs (miRs) are broad regulators of mRNA and protein expression which have been previously associated with cardiovascular aging but have not yet been implicated in SMC contractile function (16). To explore global mechanisms contributing to vascular dysfunction with aging, miR expression profiles were determined in aortic RNA from young (3-4 months) and aged (12 months) mice. To distinguish specific miRs that may contribute to vascular aging, the aging $\mathrm{miR}$ expression profile was determined in tamoxifeninduced MR-floxed, Cre-negative mice (hereafter termed "MR-intact"), which display the vascular aging phenotype, and compared to MR-floxed, Cre-positive littermates (hereafter termed "SMC-MR-KO"), which are protected from rising BP and vasoconstriction with aging. Using strict criteria of a 2.0 fold change and $P<0.01,108 \mathrm{miRs}$ were identified that changed significantly with aging in MR-intact mice and 59 aging-related vascular miRs were identified in SMC-MR-KO mice (Supplemental Table 1 and 2; supplemental material available online with this article; doi:10.1172/jci.insight.88942DS1). In both genotypes, 90 percent of the altered miRs decreased with aging while only $10 \%$ increased (Figure $1 \mathrm{~A}$ ). Of the miRs that change with aging in MR-intact mice, sixty three percent of the down-regulated miRs and $73 \%$ of up-regulated miRs, did not change with aging in the SMC-MR-KO mice and hence were considered as candidates to mediate the vascular aging phenotype (Figure 1B). Figure $1 \mathrm{C}$ displays the top 5 most downregulated (left panel) and up-regulated (right panel) miRNAs with aging in MR-intact mice compared with 

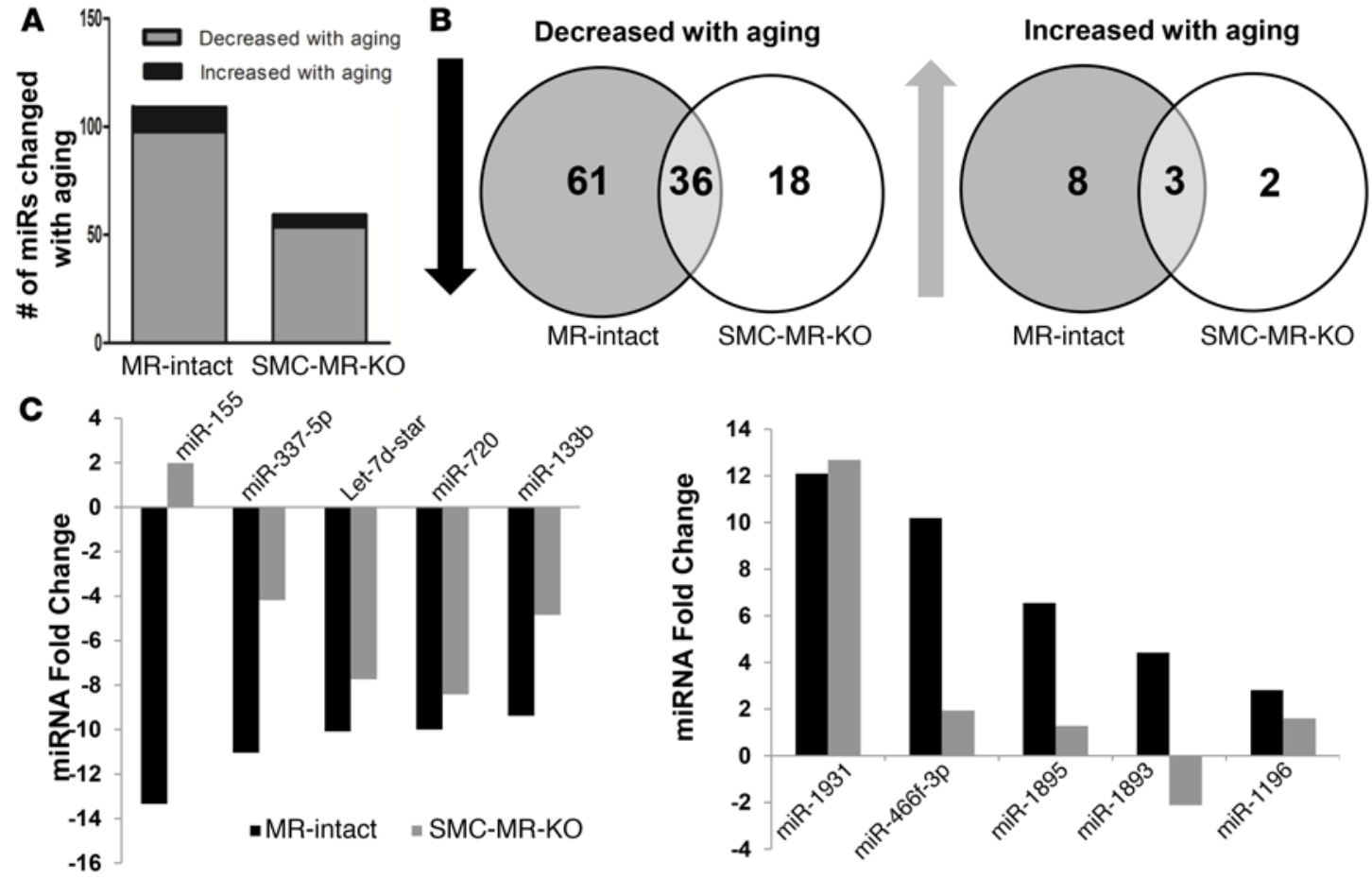

Figure 1. micro-RNA expression predominantly decreases in aging aortas and is modulated by MR in smooth muscle. MicroRNA expression was compared in aortas from young (3 months) and aged (12 months) mineralocorticoid receptor (MR)intact and smooth muscle cell mineralocorticoid receptor knock out (SMC-MR-KO) mice. (A) The total number of altered miRNAs (2.0 fold change, $P<0.01$ ) with aging from MR-intact and SMC-MR-KO mice, (black bar = increased with aging, grey bar = decreased with aging). (B) Venn diagram indicating overlap of down-regulated/up-regulated miRNAs with aging in MR-intact and SMC-MR-KO mice. (C) Top 5 down-regulated (left) and up-regulated (right) miRNAs with aging in MR-intact aortas compared with the change with aging in SMC-MR-KO aortas.

the relative change with aging in SMC-MR-KO mice. We focused on down-regulated miRs as those predominated with vascular aging and further pursued miR-155, because it is the most highly down-regulated miRNA with aging in MR-intact mice but does not decrease with aging in SMC-MR-KO mice, suggesting that it could contribute to the vascular aging phenotype. Also, in humans, miR-155 expression was previously shown to decline with aging in peripheral blood mononuclear cells (17).

Mineralocorticoid receptor increases with aging in resistance vessels and regulates miR-155. The aorta, a large conduit vessel, was used for miR profiling for practical reasons. However, it is vasoconstriction of resistance vessels that contributes to BP control. Indeed, we previously demonstrated that resistance vessel constriction in response to AngII (and other agonists) is enhanced with aging in MR-intact mice but not in aged SMC-MR-KO mice (15). Thus, we measured MR and miR-155 mRNA abundance in aging mesenteric resistance vessels. MR mRNA expression increases with aging in mouse mesenteric vessels (Figure 2A), as previously demonstrated by others in rat aortic SMCs (18). As expected, MR expression is significantly decreased in resistance vessels from both young and aged SMC-MR-KO mice. Also, vascular MR expression did not change with aging in SMC-MR-KO mice, supporting that the rise with age in the MR-intact vessels is likely due to changes in MR expression in the SMCs. miR-155 expression decreased profoundly with aging in mesenteric vessels from MR-intact mice, as in the aortic tissue microarray. This decline with aging was substantially attenuated in SMC-MR-KO mice resulting in significantly higher miR-155 expression in mesenteric vessels from aged SMC-MR-KO compared to MR-intact mice (Figure 2B). To investigate whether MR might regulate miR-155 at the level of processing versus transcription, we measured mesenteric vessel expression of unprocessed precursor miR-155 transcript and observed the same pattern, with a significant decline with aging that appears to be attenuated by SMC-MR deletion (Figure 2C). This data suggests that MR may regulate miR-155 at the level of transcription. To test this, HEK293 cells lacking endogenous MR were transfected with the miR-155 host-gene promoter driving a luciferase reporter along with a dose escalation of full length human MR expression plasmid. MR repressed miR-155 promoter 

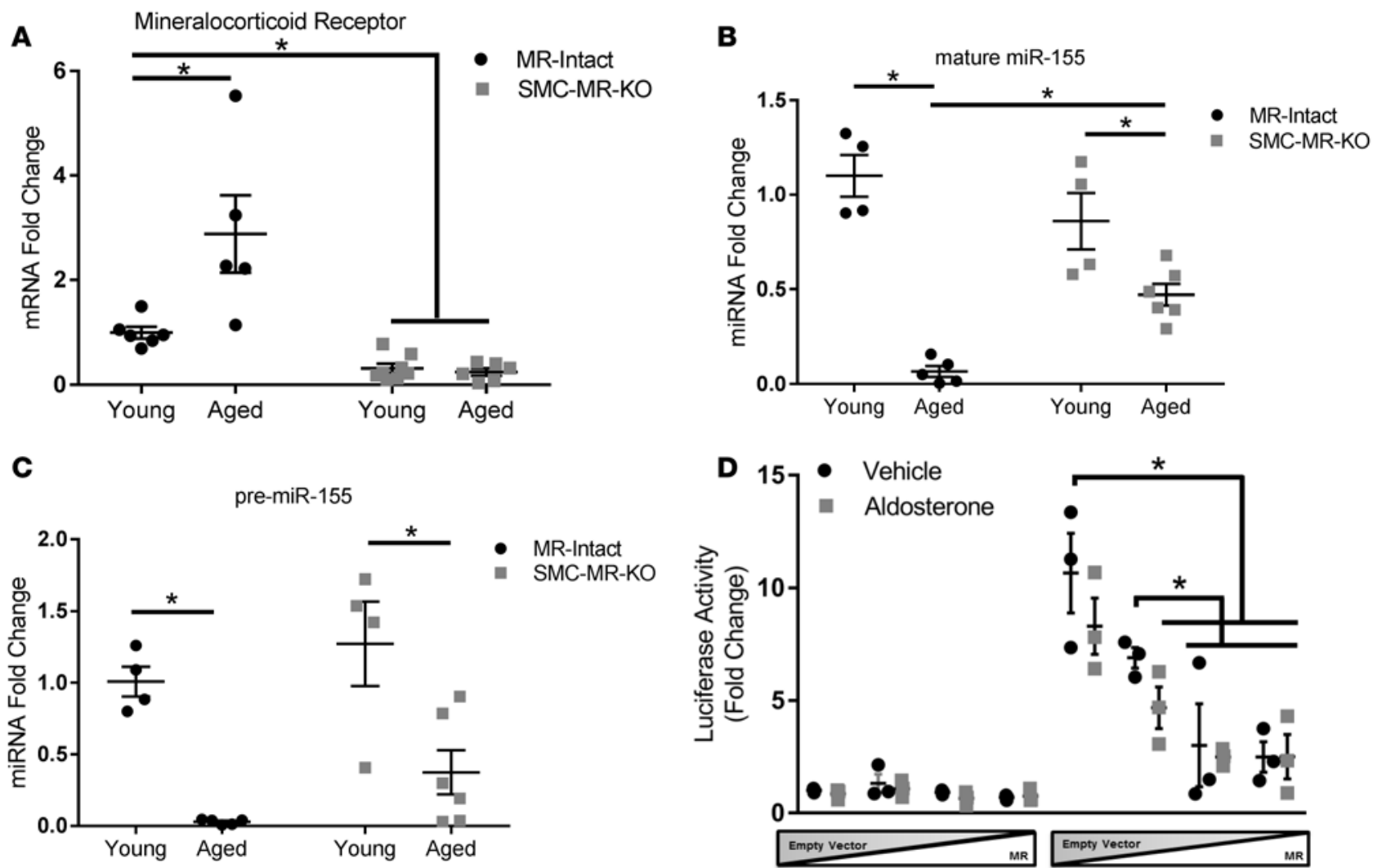

Figure 2. Mineralocorticoid receptor increases in aging resistance vessels and suppresses miR-155 transcription. Mesenteric resistance vessel mRNA expression of (A) mineralocorticoid receptor (MR), (B) mature miR-155, and (C) pre-cursor miR-155 was measured in young and aged MR-intact and smooth muscle cell mineralocorticoid receptor knock out (SMC-MR-KO) mice. (D) MR represses miR-155 host gene promoter activity. HEK293 cells were co-transfected with the miR-155 host gene promoter and increasing doses of a full length human MR expression vector. Cells were stimulated with either vehicle or $10 \mathrm{nM}$ aldosterone. (A) $\mathrm{n}=6$ young MR-intact, 5 aged MR-intact, 8 young SMC-MR-KO, 6 aged SMC-MR-KO. (B and C) n=4 young mice/group, 5 aged MR-intact, 6 aged SMC-MR-KO, (D) average of 3 independent experiments performed in triplicate. ${ }^{*} P<0.05$, Two-way ANOVA with Tukey post hoc testing for Panels A-D. Data are means \pm SEM.

activity in a dose dependent manner that was independent of the addition of the MR ligand, aldosterone (Figure 2D). Simultaneous control studies with an aldosterone-responsive promoter confirmed the activity of the ligand (Supplemental Figure 1). Together these data support a model in which MR rises with age in resistance vessel SMCs and inhibits miR-155 transcription in a ligand-independent manner.

miR-155 regulates the L-type Calcium Channel (LTCC) and Angiotensin II type-1 receptor (AgtR1) in Mesenteric $S M C$ s. Two approaches were used to identify potential SMC miR-155 target genes that could contribute to vascular aging. Using a candidate target gene approach, we examined AgtR1, since miR-155 has been previously shown to target the AgtR1 in human and rat cells $(19,20)$ and we previously demonstrated that vascular AngII signaling is attenuated in aged SMC-MR-KO mice (15). An unbiased target filter analysis was also performed using Ingenuity Pathway Analysis to search for predicted miR-155 target genes that are known to be involved in cardiovascular signaling. This analysis identified CACNA1C (Cav1.2), the poreforming LTCC subunit, as a potential novel miR-155 target gene. To test these predicted targets, miR-155 was transfected into mouse mesenteric SMCs to produce a significant increase in SMC miR-155 (Figure 3A). Transfection of miR-155 resulted in a significant decrease in mRNA for Cav1.2 and AgtR1b (the predominant AgtR1 in mouse mesenteric vessels) compared to scrambled control miR (Figure 3B). miR-155 overexpression did not alter $\alpha 2 \delta$-1, another LTCC subunit involved in channel membrane localization, or AgtR2, the vasodilatory angiotensin receptor, supporting the specificity of these targets (Supplemental Figure 2). Similarly, overexpression of miR-155 in aortic SMC decreased expression of Cav1.2 and AgtR1a (the predominant AgtR1 expressed in mouse aorta (21), Supplemental Figure 3). In whole mesenteric vessels from MR-intact mice, Cav1.2 and AgtR1b mRNA increase with aging (Figure 3, C and D) in association with a decline in miR-155 (Figure 2A). Conversely, in mesenteric vessels from aged SMC-MR-KO 
A

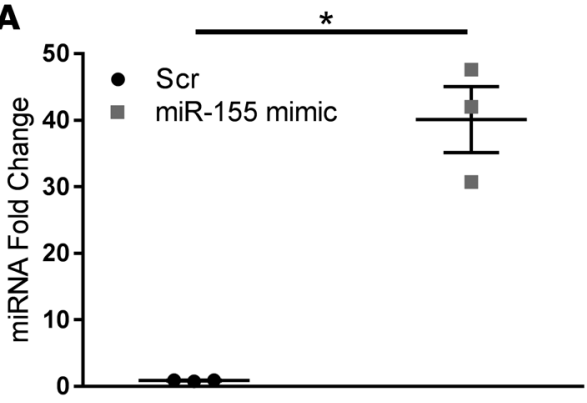

C

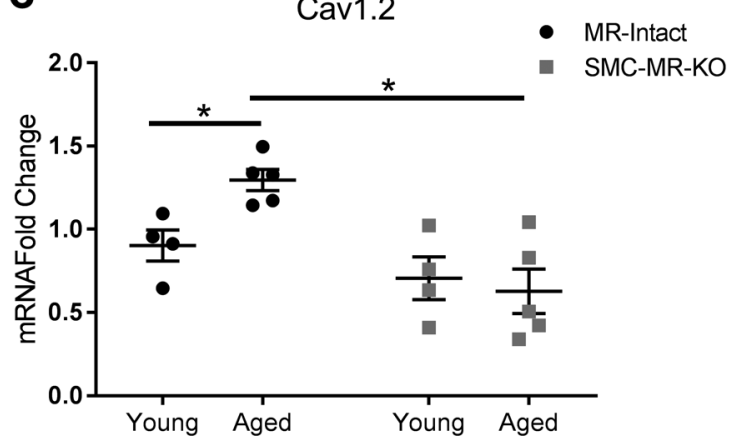

B

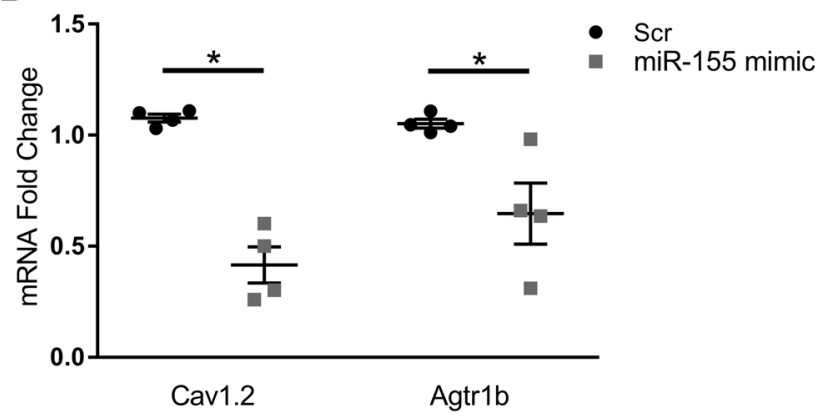

D Agtr1b

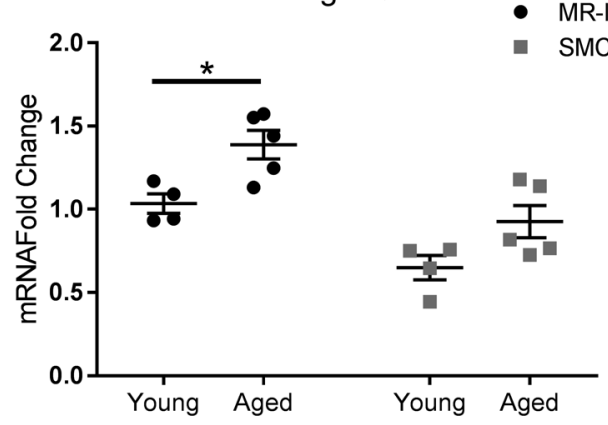

Figure 3. miR-155 expression inversely correlates with L-type calcium channel and angiotensin II type 1 receptor expression in mesenteric smooth muscle cells and vessels. Mouse mesenteric resistance vessel smooth muscle cells were transfected with either scrambled control miR (Scr) or miR-155 mimic in full serum for 48 hours. (A) miR-155 expression and (B) target gene mRNA expression of Cav1.2 (the pore forming subunit of the L-type calcium channel) and angiotensin II type 1b receptor (Agtr1b) was quantified in transfected cells. Mesenteric resistance vessel mRNA expression of (C) Cav1.2 and (D) Agtr1b was quantified in young and aged mineralocorticoid receptor (MR)-intact and smooth muscle cell mineralocorticoid receptor knock out (SMC-MR-KO) mice. (A and B) $n=3$ independent experiments performed in triplicate (Unpaired two-tailed student's $t$ tests). (C and $\mathbf{D}) \mathrm{n}=4$ young mice/group, 5 aged mice/ group. ${ }^{*} P<0.05$. (Two-way ANOVA with Tukey post hoc testing). Data are means $\pm \mathrm{SEM}$.

mice in which miR-155 is increased compared to aged MR-intact littermates (Figure 2A), Cav1.2 mRNA is significantly decreased compared to aged MR-intact vessels (Figure 3C). The data thus far support the concept that SMC-MR transcriptionally represses miR-155 in aging resistance vessels resulting in increased vascular Cav1.2 and AgtR1 expression.

SMC-MR contributes to BP, resistance vessel tone, SMC LTCC Function, and LTCC-mediated vasoconstriction with aging. We previously demonstrated that SMC-MR-KO mice are protected from the rise in $\mathrm{BP}$ from 7 to 10 months of age with no change in aldosterone levels, renal function or the sodium responsiveness of BP (15). Here we confirm that there is no difference in BP between genotypes in another small cohort of 3 month old mice (Figure 4A and Supplemental Figure 4). Systolic BP rises modestly but significantly with aging from 3 to 12 month of age in MR-intact mice but not in SMC-MR-KO littermates. The vasculature contributes to rising $\mathrm{BP}$ by constricting in response to increased blood volume or to contractile agonists. This vasoconstriction is mediated by activation of LTCCs in SMCs resulting in vascular calcium influx which induces global release of intracellular calcium stores and results in vasoconstriction (22). This contractile response of resistance vessels to increasing intraluminal pressure is termed myogenic tone. Here we show that in young mice, the presence of SMC-MR did not affect myogenic tone. Although mesenteric vessel tone did not change with aging in MRintact mice, it was significantly decreased in aged SMC-MR-KO mice compared to aged MR-intact mice (Figure 4B). Patch clamp studies in freshly dispersed mesenteric SMCs similarly showed that in young mice, the presence of SMC-MR does not affect basal SMC calcium current or LTCC-specific current (stimulated with BayK-8644, Figure 4C, left). However, mesenteric SMC from aged SMC-MR-KO mice had decreased basal and BayK-activated calcium current compared to MR-intact controls (Figure 4C, right). The LTCC blocker nifedipine eliminated the currents, confirming that these are indeed LTCC-specific (Supplemental Figure 5). Fura-2 photometric measurement of LTCC-activated calcium influx into mesenteric arteries similarly revealed no difference in young mice, while aged MR-intact mice exhibited increased calcium influx compared to SMCMR-KO littermates (Figure 4D). Finally, when mesenteric vessel contractile force was measured in response to 
A
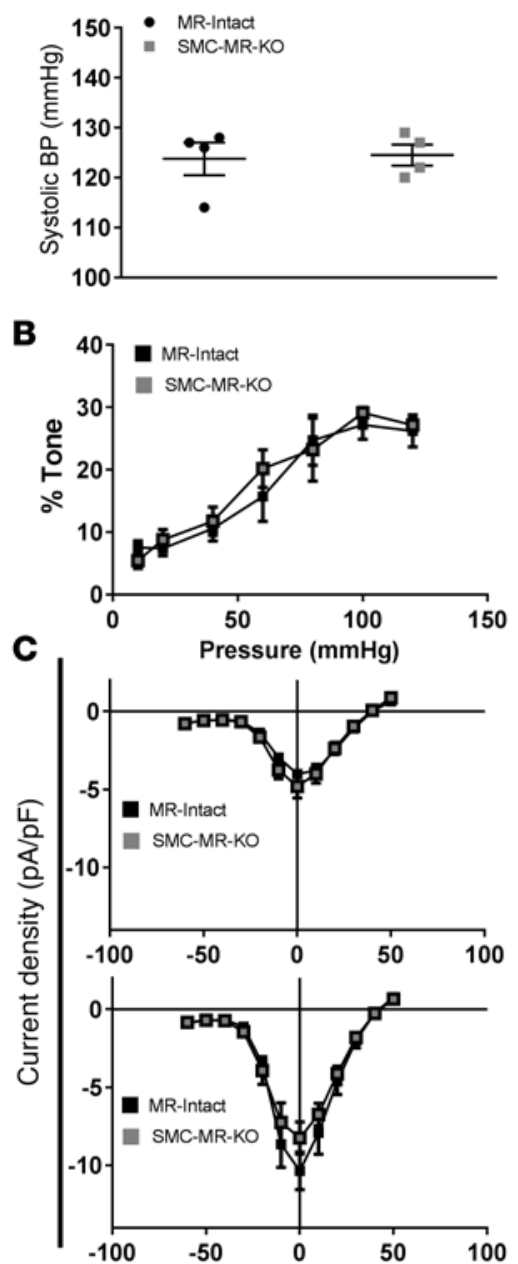

D

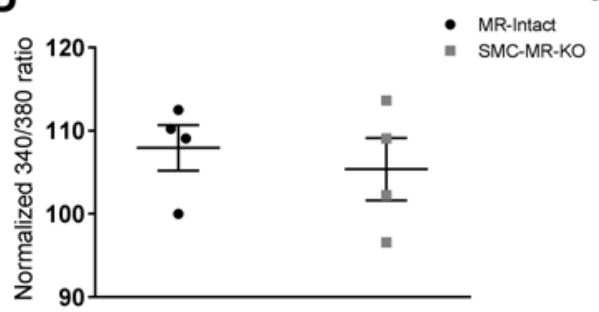

E

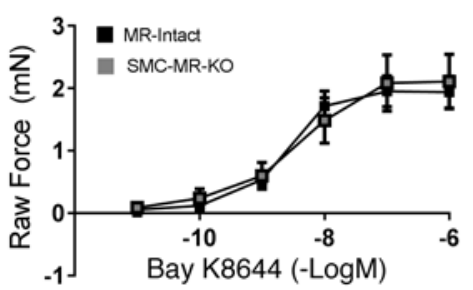

Aged
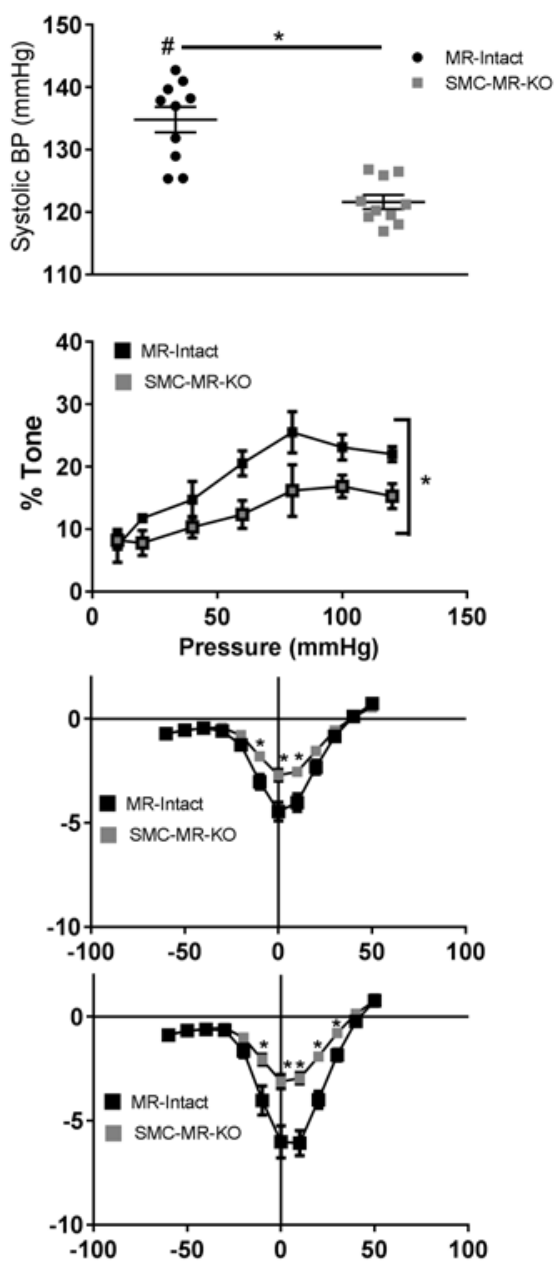

Voltage (mV)
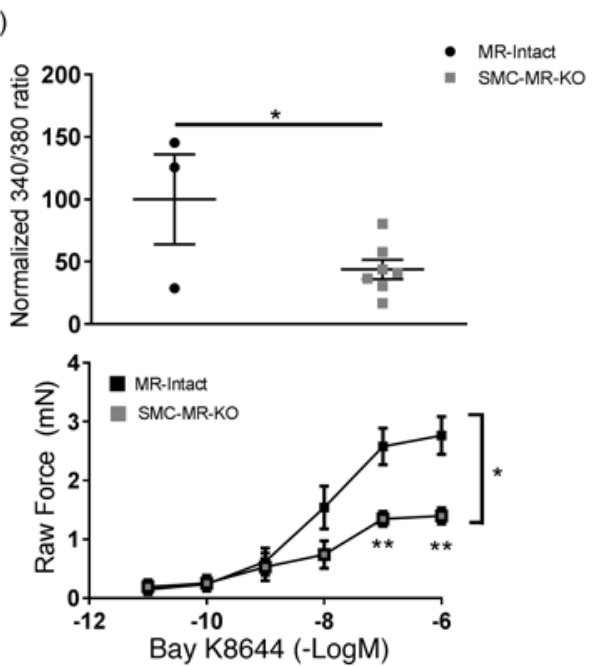

Figure 4. SMC-MR contributes to BP control, myogenic tone, vascular L-type calcium channel activity, and vasoconstriction with aging. (A)

Mean arterial pressure measured via arterial telemetry devices in young ( $n=4 /$ group) and aged mineralocorticoid receptor (MR)-intact $(n=10)$ and smooth muscle cell mineralocorticoid receptor knock out (SMC-MRKO) mice $(n=10)$. (B) Myogenic tone responses of mesenteric resistance vessels over a range of intraluminal pressures in young and aged MRintact and SMC-MR-KO mice $(\mathrm{n}=4$ young mice/group, $n=3$ aged mice/ group). (c) Patch clamp studies of freshly dispersed mesenteric resistance vessel SMC from young and aged MR-intact and SMC-MR$K O$ mice were performed and current density was measured under basal conditions (Top panels) and with the L-type calcium channel (LTCC) agonist BayK-8644 (Bottom panels). $\mathrm{n}=$ $16-18$ cells, from 3 young mice/group or 4 aged mice/group. (D) Whole mesenteric vessel calcium flux was measured in response to BayK8644 via Fura-2 photometry. Fluorescent emission $(510 \mathrm{~nm})$ was quantified and expressed as the change in the $340 / 380$ ratio in response to LTCC activation with BayK8644, $(n=4$ young mice/group, 3 aged MR-intact mice/group, and 7 aged SMC-MR-KO mice/group). (E) Mesenteric vessel contractile responses to LTCC agonist BayK-8644 in young and aged MR-intact and SMC-MR-KO mice, ( $\mathrm{n}=5$ mice/group). ${ }^{*} P<0.05,{ }^{* *} P$ $<0.05$ at each specific dose, $\# P<$ 0.05 vs. young MR-intact. Two-way repeated measures ANOVA with Tukey post hoc testing for panels A-E. Data are means \pm SEM.

increasing doses of LTCC agonist, there was no difference in young mice lacking SMC-MR, while aged SMCMR-KO mice had significantly decreased LTCC-mediated contraction (Figure 4E). Together, these data support the concept that SMC-MR is necessary to maintain LTCC activity in the aging vasculature and that this may prime the aged vasculature for enhanced vasoconstriction in response to renal-mediated sodium retention and/or stimulation by contractile agonists, both of which are increased with aging in humans. 
Restoration of miR-155 in SMCs rescues the vascular aging phenotype in aged mice. Since aged MR-intact mice have decreased miR-155 compared to SMC-MR-KO mice and this is associated with increased LTCCmediated constriction and AngII-induced vasoconstriction and oxidative stress, we next examined whether restoration of SMC miR-155 in aged MR-intact mice could rescue this vascular aging phenotype. Aged MR-intact mice were injected with a lentivirus specifically targeting miR-155 expression to SMC. These mice were compared to aged MR-intact or SMC-MR-KO mice injected with a control lentivirus. Injection of miR-155 lentivirus significantly increased aortic miR-155 expression without changing aortic MR mRNA expression (Supplemental Figure 6) or miR-155 levels in tissues that do not contain a substantial contribution of SMCs (Supplemental Figure 7). Restoration of miR-155 levels in the aorta was associated with decreased expression of the miR-155 target genes, Cav1.2 and AgtR1a, to levels that are not different from aged SMC-MR-KO mice (Figure 5, A and B). Treatment with lentivirus to augment SMC miR-155 levels in aged vessels was associated with reduced LTCC and AngII-mediated vasoconstriction in MR-intact mice to achieve the same degree of constriction as SMC-MR-KO mice (Figure 5, C and D). Treatment with miR-155 lentivirus did not affect vasoconstriction to the adrenergic agonist phenylephrine (Supplemental Figure 8) supporting the specificity of this effect. Thus the vasoconstriction phenotype in aged resistance vessels induced by SMC miR-155 expression is similar to the phenotype induced by deletion of SMC-MR, with protection from enhanced AngII vasoconstriction with age without attenuating the rise in vasoconstriction to phenylephrine (15). Furthermore, miR-155 treatment prevented AngII-induced oxidative stress in carotid arteries (Figure 5, E and F), an important component of vascular aging (11) that is also prevented in SMC-MR-KO mice treated with control virus.

Serum miR-155 may predict the BP-Lowering response to MR antagonist therapy in aged humans. Based on the mechanism elucidated in mice, we considered whether MR antagonism in aging humans may lower $\mathrm{BP}$ in part by suppressing miR-155 expression. To begin to address this, miR-155 levels were measured in serum from 16 otherwise healthy older adults ( 8 Male, 8 Female, ages 55-79) who had participated in a one month, randomized, blinded, crossover trial of the MR antagonist eplerenone (Supplemental Table 3) (23). In this small group of older adults, there was substantial variability between subjects in the serum miR-155 response to MR antagonist treatment (Supplemental Figure 9). Based on this finding, the cohort was divided into two groups to compare subjects in whom serum miR-155 increased during MR blockade $(\mathrm{N}=7)$ to those with no increase with eplerenone treatment $(\mathrm{N}=9)$. Subjects in whom serum miR-155 levels increased after MR inhibition had a significantly greater decline in both systolic and diastolic BP in response to eplerenone treatment (Figures 6, A and B). To determine if the baseline level of miR-155 might predict the BP response to MR antagonism, the basal serum miR-155 value was correlated with the change in BP after 1 month of eplerenone (Figures 6, C and D). The baseline serum level of miR-155 significantly correlated with the change in diastolic BP in response to MR blockade (Figure 6D). Although the study is small, these data support the concepts that in older adults, lower serum miR-155 may identify a subgroup more likely to benefit from MR inhibition to lower their BP, and that MR inhibition may lower BP in part by raising miR-155. Additional studies are needed to confirm these findings in larger cohorts and in patients with significant systolic hypertension to determine whether miR155 may have clinical benefit as a biomarker to tailor antihypertensive therapy in the elderly.

\section{Discussion}

In summary, by comparing aging MR-intact mice to SMC-MR-KO littermates, we identified molecular mechanisms contributing to vasoconstriction with aging. We demonstrated that: (1) There is a substantial decline with aging in expression of vascular miRs and identified miR-155 as the most profoundly decreased vascular miR in aging mouse vessels, but not when MR is deleted from SMCs; (2) MR expression increases with aging in mesenteric resistance vessels and MR transcriptionally suppresses the miR-155 host gene promoter in SMCs, independent of its ligand aldosterone; (3) Aged MR-intact mice with decreased vascular miR-155 expression also have increased vascular mRNA expression of the predicted miR-155 targets Cav1.2 and AgtR1; (4) Aged SMC-MR-KO mice have decreased BP and myogenic tone in association with decreased SMC LTCC activity, mesenteric vessel calcium signaling, and LTCC-mediated vasoconstriction when compared to aged matched MR-intact littermates; (5) Restoration of miR-155 specifically in SMCs in aged MR-intact mice rescues the aging phenotype resulting in attenuated LTCC- and AngII-mediated vasoconstriction and AngII-induced vascular oxidative stress; and finally (6) In aged humans, lower serum miR-155 and rising miR-155 with treatment, predict the BP-lowering response to MR-antagonist therapy. 
A

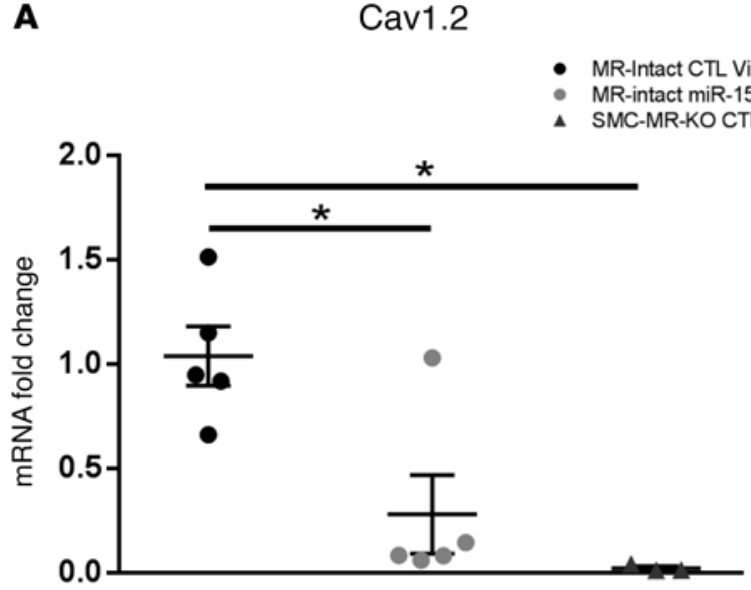

C

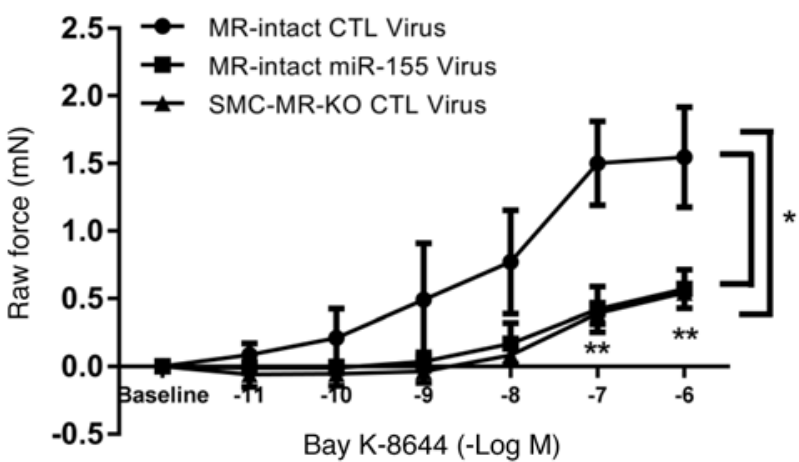

E

Vehicle

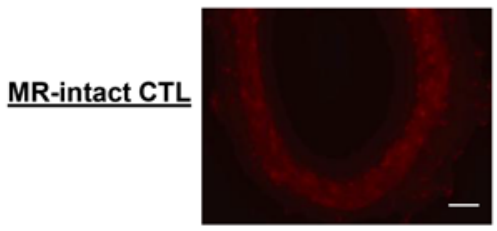

MR-intact miR-155
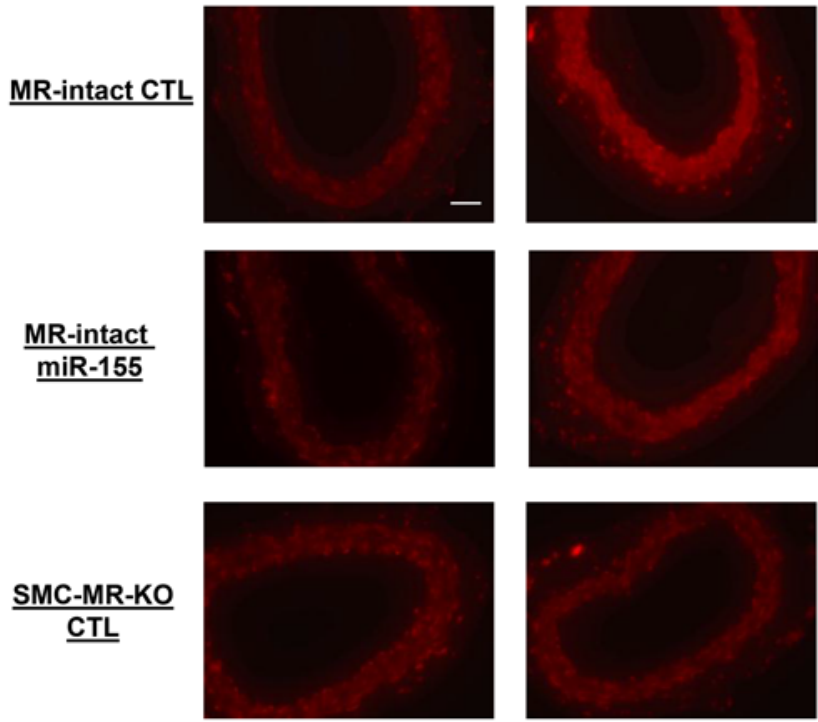

B

Agtr1a

MR-Intact CTL Virus

MR-intact miR-155 virus

SMC-MR-KO CTL Virus
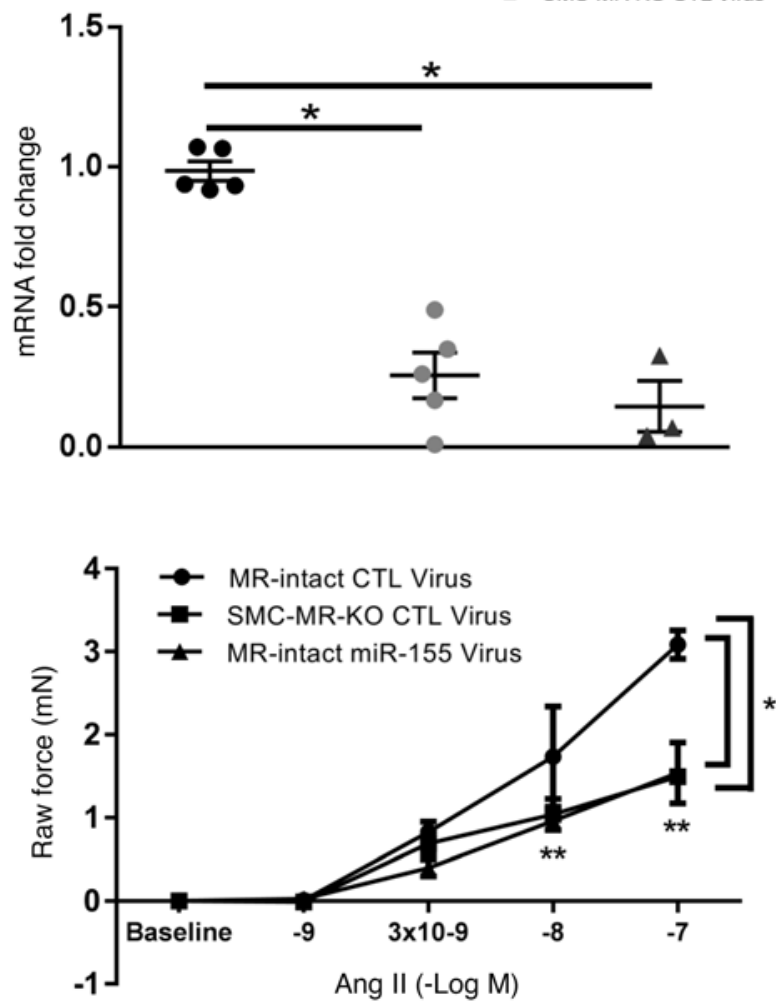

$\mathbf{F}$

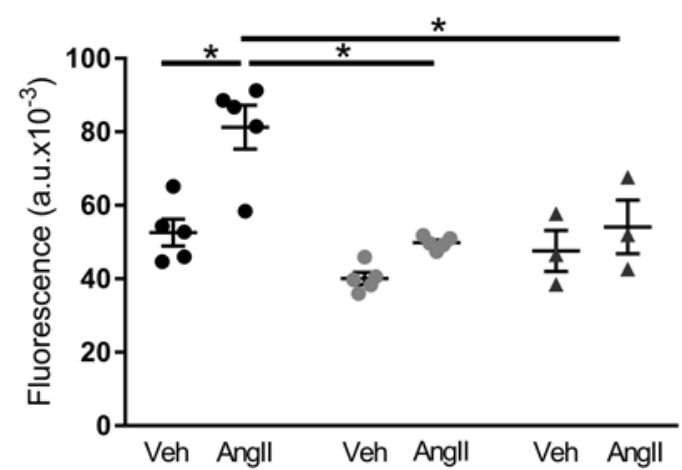

- MR-Intact CTL Virus

- MR-intact miR-155 virus

- SMC-MR-KO CTL Virus

Figure 5. Restoration of miR-155 in SMCs rescues the vascular aging phenotype in aged mice. (A-F) Aged mineralocorticoid receptor (MR)-intact mice were treated with either control $(C T L)$ virus $(n=5)$ or smooth muscle cell (SMC)-specific miR-155 expressing lentivirus (miR-155 Virus) ( $n=5)$ and compared to aged and SMC-MR-KO mice treated with the control virus $(n=3)$. Aortic miR-155 target gene expression was measured for (A) Cav1.2 and (B) Agtr1a. Mesenteric vessel contractile responses were measured by wire myography in response to (C) LTCC agonist BayK-8644 and (D) Angiotensin II (AngII). Angll-induced oxidative stress was quantified by dihydroethidium (DHE) staining; (E) Representative images of DHE stained carotid arteries treated with saline vehicle (Veh) or Angll. Scale bar $=100 \mu \mathrm{M}$. (F) Quantification of Angll-induced reactive oxygen species generation. ${ }^{*} P<0.05(\mathbf{A}-\mathbf{F})$, ${ }^{* *} P<0.05$ at each specific dose (C and D). One-Way ANOVA with Bonferroni post hoc testing ( $\mathbf{A}$ and $\mathbf{B}$ ), Two-way Repeated Measures ANOVA with Bonferroni post hoc testing (C and $\mathbf{D})$. Two-way ANOVA with Bonferroni post hoc testing (F). Data are means \pm SEM. 

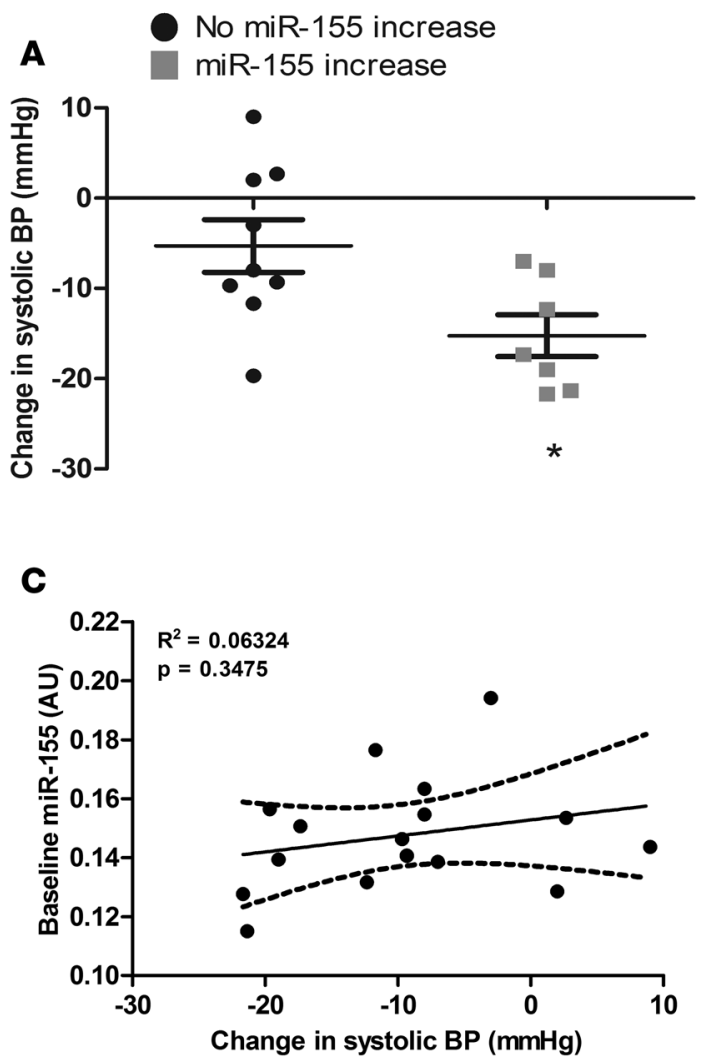

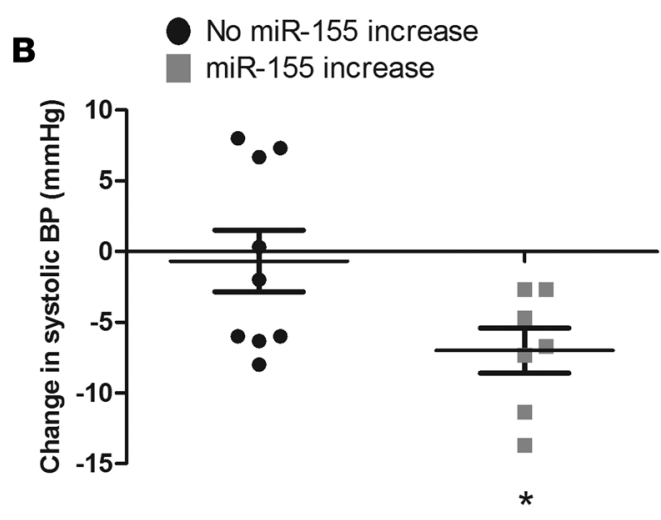

D

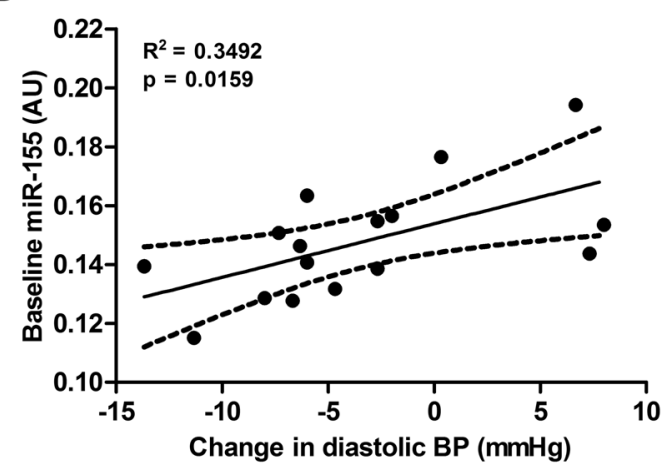

Figure 6. Serum miR-155 predicts the blood pressure lowering response to mineralocorticoid receptor antagonist therapy in aged humans. The change in (A) Systolic and (B) diastolic blood pressure in patients with an increase in serum miR-155 ( $n=7)$ after one month of eplerenone treatment was compared to those in which miR-155 did not increase with treatment $(n=9) .{ }^{*} P<0.05$, Un-paired two-tailed student's $t$ tests. Panels (C) and (D) show the correlation between baseline serum miR-155 levels versus the change in (C) systolic and (D) diastolic blood pressure with eplerenone treatment (Pearson Correlation). Data are means \pm SEM.

Overall, these data provide new insight into mechanisms driving vasoconstriction with aging that may contribute to the associated rise in BP. The data are consistent with the model in Figure 7 in which enhanced SMC-MR expression and activity in aging resistance vessels suppresses vascular miR-155 transcription resulting in increased LTCC and AgtR1 expression. In this way, SMC-MR contributes to maintenance of myogenic tone and LTCC-induced constriction and primes the vasculature for enhanced AngII-induced oxidative stress and vasoconstriction, important components of the vascular aging phenotype that contributes to hypertension with aging. These results support the need for further studies in humans to determine if miR-155 could be a biomarker of MR activation in the setting of vascular aging with important implications for improving BP control in the rapidly aging population.

Many vascular miRNAs change with aging (reviewed in 16, 24). However, these data are the first to identify miR-155 as being down-regulated in aging vessels and to describe a miR that regulates SMC contractile function in the aging vasculature. Prior studies reported that miR-155 expression is reduced in peripheral blood mononuclear cells from old compared to young humans (17). Additionally, serum miR-155 is reduced in patients with chronic kidney disease, a population heavily burdened with hypertension and cardiovascular disease (19), further supporting the potential relevance of this pathway in aging humans. We have further identified SMC-MR as a negative regulator of vascular miR-155. A recent study showed that MR also negatively regulates miR-29b in aortic SMCs by enhancing its degradation (25). Here we show that MR transcriptionally represses the miR-155 host-gene promoter in a ligand-independent manner. Thus, SMCMR appears to regulate multiple miRs in vascular SMCs by distinct mechanisms. While other studies have only identified mechanisms that activate the miR-155 promoter $(26,27)$, our results identify MR as the first negative regulator of miR-155. Further studied are needed to determine whether this mechanism contributes to other pathologies where MR activation is increased, including obesity, diabetes, and heart failure $(28,29)$.

Steroid receptors are capable of negative regulation of transcription, although the mechanisms for trans-repression are incompletely elucidated and are best studied for the highly homologous glucocorticoid receptor (30). One mechanism involves binding of steroid receptors to so called "negative response elements" in DNA where they recruit corepressors to suppress gene transcription. Another mechanism involves binding of activated steroid receptors to other DNA-bound transcription factors to suppress transcription. We did not identify a predicted MR binding site in the miR-155 host gene promoter but rather, there are 


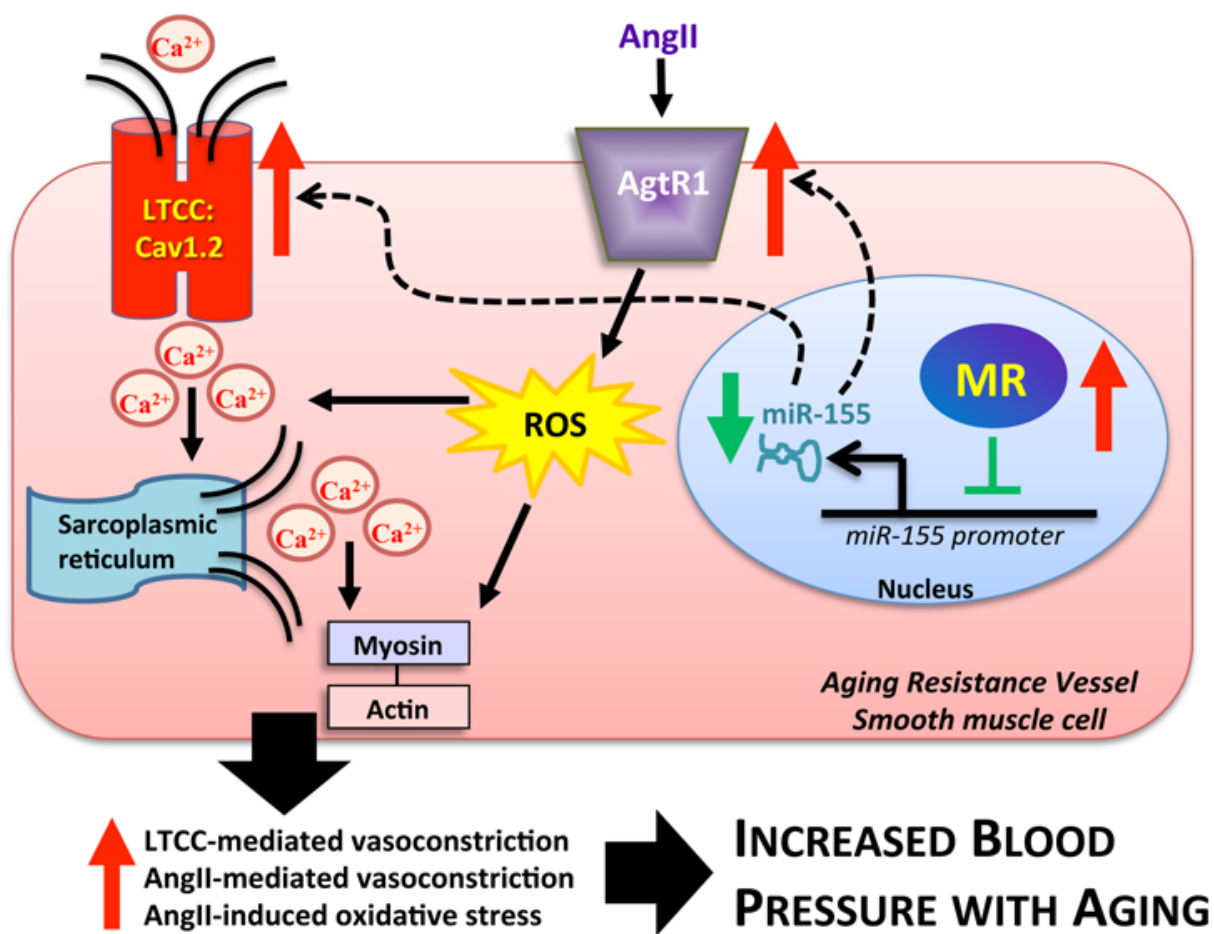

Figure 7. Model of the Role of Smooth Muscle Cell Mineralocorticoid Receptor in Vascular Aging. These data are consistent with a model in which mineralocorticoid receptor (MR) expression rises in aging resistance vessel smooth muscle cells where MR suppresses miR-155 transcription leading to the up-regulation of angiotensin type 1 receptors (AgtR1) and L-type calcium channels (LTCC). The up-regulation of these pro-constrictive genes contributes to SMC calcium influx and reactive oxygen species (ROS) production. This enhances Angll-induced and LTCC-mediated vasoconstriction and vascular oxidative stress, components of vascular aging. Restoration of miR-155 in SMC in aging vessels reverses these changes with aging. These aging-induced alterations in vascular function ultimately contribute to increased blood pressure with aging. MR antagonists may decrease blood pressure in part by inhibiting this mechanism in the aging vasculature. Cav1.2 = voltage gated subunit of the L-type calcium channel, $\mathrm{Ca}^{2+}=$ calcium, AngII = angiotensin II.

known activating sites for the transcription factors $\mathrm{AP} 1$ and $\mathrm{NFkB}$, two transcriptional regulators that are known to be modulated by steroid receptors including the MR $(31,32)$. Our data suggest that SMC-MR expression rises with aging and suppresses miR-155 promoter activity in a ligand-independent manner. The mechanism by which MR increases with aging is not known, but MR can be activated independent of ligand by the Rho-family small GTPase Rac1 (33) or by direct signaling downstream of the AgtR1 (14). Future studies will determine the detailed molecular mechanism by which MR is activated and regulates miR-155 in aging SMCs.

Our data support that miR-155 may contribute to vasoconstriction in the aging vasculature by regulating expression of LTCCs and angiotensin type-1 receptors. We demonstrate that in MR-intact vessels, decreased miR-155 with aging is associated with increased vascular Cav1.2 and AgtR1 mRNA expression and that overexpression of miR-155 in cultured SMCs decreased expression of these genes. Furthermore, restoration of SMC miR-155 in the aging vasculature in vivo decreases Cav1.2 and AgtR1 mRNA expression and attenuates LTCC- and AngII-mediated vasoconstriction. These data extend previous studies demonstrating that miR-155 targets the AtgR1 in human and rat cells $(19,20)$. Interestingly, a single nucleotide polymorphism in the human AtgR1 3'-untranslated region has been identified that prevents miR-155 binding resulting in increased AtgR1 expression. This polymorphisms is associated with hypertension in humans (34), further supporting a role for miR-155 regulation of AtgR1 in human hypertension.

In animal models of hypertension, systemic vascular resistance is increased due to enhanced vasoconstriction mediated by LTCC-dependent calcium entry into SMC $(35,36)$. Indeed, mesenteric arterioles of spontaneously hypertensive rats (SHR) have increased myogenic responsiveness, abnormal calcium-dependent vascular tone (37-39) and increased Cav1.2 mRNA expression (35). Vascular SMCs from 
SHR similarly have elevated whole-cell and single-channel LTCC currents and enhanced calcium influx versus normotensive controls (40-43). Here we show that in the absence of any hypertensive stimuli, aging mice also have increased vascular Cav1.2 mRNA expression and that this may be mediated by SMC-MR regulation of miR-155, a novel regulator of Cav1.2. Future studies are needed to determine if this SMC-MR/miR-155-mediated regulatory mechanism also contributes to LTCC regulation in animal models of hypertension.

It is important to note that despite the rise in Cav1.2 mRNA in aging MR-intact resistance vessels, myogenic tone and LTCC current density are not increased with aging but rather are decreased in aged SMCMR-KO vessels in which miR-155 expression is relatively preserved and Cav1.2 is decreased. In addition to Cav1.2 mRNA levels, other factors contribute to LTCC function including protein levels of Cav1.2 and other LTCC subunits, localization of LTCCs to the membrane, and factors that modulate channel function independent of channel number, including oxidative stress. Myogenic tone is also modulated by other channels (although we have previously demonstrated that SMC-MR deletion does not alter Ca-activated potassium channel mRNA expression or function in this animal model (15)). Some of these other factors are also likely modified by aging and deserve further study. Thus, the changes in LTCC mRNA expression do not completely explain the vascular tone and BP phenotype in these mice. Rather, the data support the concept that SMC-MR is necessary for maintenance of myogenic tone with aging thereby priming the vasculature for enhanced vasoconstriction in response to vasoconstrictive factors that increase with aging in mice including AngII signaling and oxidative stress, and may also enhance the response to renal-mediated sodium retention that occurs in aging humans. Together, these mechanisms may contribute to rising blood pressure with age and may explain why aged mice lacking SMC-MR have lower BP.

The findings that vascular miR-155 is profoundly decreased with aging, regulates genes that contribute to vasoconstriction, and can be restored in SMCs of the aging vasculature to reverse the aging phenotype, raises the possibility that SMC miR-155 could be a therapeutic target to reverse vascular aging and potentially improve BP control the elderly. Future studies are also warranted to determine whether miR-155 contributes to other known aging mechanisms such as vascular cell senescence or autophagy. However, it is critical to note that miR-155 is expressed in many other cell types where it is well studied and has varied functions. While our studies focus on SMCs, in endothelial cells (EC), miR-155 targets endothelial nitric oxide synthase, a vasodilatory enzyme (44), as well as other pathways involved in vascular EC dysfunction (45). Thus, while raising miR-155 in aging SMC may be beneficial for vascular function, raising miR-155 in endothelial cells may have opposing effects. miR-155 is also well studied in cancer, both as a biomarker and as a direct driver of B-cell and other hematologic malignancies (45). Thus, any attempts to enhance miR155 as an anti-aging or anti-hypertensive therapy would need to be very specific to SMCs to avoid adverse effects on EC function $(44,46)$ and to avoid hematologic cancers or autoimmune disorders due to miR-155 effects in leukocytes. Methods for cell-type specific delivery of nucleic acids using targeted nanoparticles are already in development (47-50) and thus such specific therapies could hold promise in the future. Conversely, these data suggest caution in developing systemic anti-miR-155 therapies to treat cancer, as this might accelerate vascular SMC aging pathology. In the short term, MR antagonist drugs might be used to modulate SMC function to prevent vasoconstriction with aging. The MR antagonists spironolactone and eplerenone are already approved for treatment of hypertension and heart failure and a novel class of nonsteroidal MR antagonists is currently in clinical trials. Future studies in vitro and in mice are needed to examine whether MR inhibition directly suppresses SMC miR-155 expression as a potential novel mechanism for their efficacy. Although MR antagonist drugs lower BP and decrease cardiovascular mortality (4), their use is often limited, particularly in the elderly, due to concerns about hyperkalemia from renal MR inhibition (51). The current trial-and-error approach to hypertension therapy has not been very effective in the elderly, as clinical data shows poor BP control in this population. Our small clinical study supports the hypothesis that lower serum miR-155 may identify a subgroup of older individuals with enhanced SMCMR activation and hence may derive greater benefit from MR inhibition to lower BP. However, our study includes a very small cohort of otherwise healthy older individuals and hence has several limitations. In addition to the small sample size, the patients did not have substantial hypertension and hence may have been selected for those with low MR activation. This may contribute to the lack of correlation between basal serum miR-155 level and the decline in systolic BP. This is important as the elderly develop predominantly systolic hypertension. These limitations support the initiation of larger studies to explore the utility of serum miR-155 as a biomarker to predict the BP response to MR antagonists in elderly hypertensives 
and in patients with resistant hypertension, a population in which MR inhibition is particularly effective (52). Effects of other antihypertensive drugs on miR-155 levels should also be examined to determine the specificity of this mechanism. Such precision medicine strategies have recently been applied successfully to cancer therapy and could revolutionize the treatment of hypertension.

Several limitations to this study must be acknowledged. First, for practical reasons mouse aorta was used for microRNA profiling. Nevertheless, we discovered that miR-155 is profoundly down-regulated in the aging aorta and confirmed a similar expression pattern in aging mesenteric vessels that contribute to vascular resistance. Other miRs identified in the aorta will need to be examined in resistance vessels to determine if they might contribute to hypertension. In addition, since antibodies that recognize MR or AtgR1 are not specific in mouse tissues (53), only mRNA and not protein levels are shown. Additional measures of vascular oxidative stress should be examined in the future as DHE staining is not completely specific. Also for practical reasons, these studies were performed only in male mice. As substantial evidence supports sex differences in vascular aging pathology and BP control in the elderly $(54,55)$, future studies will determine if the novel mechanism reported here in male mice also contributes to vascular aging in females. It is important to note that although systolic BP rises modestly with aging in mice, wild type C57B16 mice do not develop frank hypertension as they age and hence we could not directly test whether the SMCmiR-155 lentivirus could be a treatment for hypertension. Rather, our findings indicate an important role for SMC-MR regulation of miR-155 in the vascular aging phenotype including enhanced vasoconstriction, oxidative stress, and AngII signaling $(1,11)$. These vascular aging mechanisms contribute to hypertension in aging humans in the setting of other factors not present in healthy mice including chronic high sodium intake, obesity, genetic factors and atherosclerotic vascular disease. Future studies will be needed to explore the role of this pathway in hypertensive animal models and in human hypertension. Finally, the clinical study reported here has many limitations inherent in human trials, and specifically is limited by very small sample size. Despite this limitation, the strengths of the randomized, blinded, crossover design resulted in detection of basal and treatment-associated changes in serum miR-155 as significant predictors of BP response to MR inhibition. Larger clinical studies are surely necessary to clarify the potential role of serum miR-155 as a biomarker to choose or monitor anti-hypertensive therapy.

Acknowledging these limitations, these data have several translational implications. Given the recent results of the SPRINT trial $(7,8)$, there is a critical need for better BP control in older individuals. This new mechanistic understanding linking SMC-MR, calcium channels, and angiotensin receptors to the pathogenesis of elevated BP with aging supports testing of novel anti-hypertensive drug combinations to improve BP control in the elderly. For example low dose of MR antagonist (or AgtR1 blocker) combined with a low dose calcium channel blocker may be particularly effective by blocking two sequential steps in this newly identified pathway that may drive hypertension in the elderly without synergistically raising serum potassium. In addition, in our small clinical study, basal serum miR-155 levels and the change with MR inhibition in aged humans, seemed to predict the BP lowering response to eplerenone. MR antagonists are potent anti-hypertensives in subsets of patient that currently are identified empirically $(56,57)$. However, the small but concerning risk of hyperkalemia limits the widespread use of MR antagonists in all elderly hypertensives to identify this subset of strong responders. If reproduced in larger studies of hypertensive patients, perhaps this approach could lead to new precision medicine strategies for hypertension management in the elderly. Finally, the lentiviral rescue study provides pre-clinical evidence supporting that restoring vascular SMC miR-155 levels could reverse the vascular aging phenotype. As targeted nanoparticles and other novel approaches are developed to deliver drugs to specific cell types, this may offer a novel therapeutic strategy to prevent the adverse cardiovascular complications of aging. In summary, we provide data supporting a novel mechanism contributing to vasoconstriction in the aging vasculature in which SMC-MR suppression of miR-155 enhances LTCC and AngII signaling in resistance vessels. This insight provides novel strategies to address the critical need for improved BP control in the elderly in order to prevent the associated cardiovascular morbidity and mortality.

\section{Methods}

Reagents and cell lines. Aldosterone (Sigma-Aldrich) was dissolved in with dimethylsulfoxide (SigmaAldrich) as described (14). BayK8644 (Sigma-Aldrich) and nifedipine (Sigma-Aldrich) were dissolved in $100 \%$ ethanol. Ang II (Sigma-Aldrich), Phenylephrine (Sigma-Aldrich), and Acetylcholine (Sigma- 
Aldrich) were dissolved in deionized $\mathrm{H}_{2} \mathrm{O}$. Appropriate vehicle controls were used in all studies. Human embryonic kidney-293 (HEK293) cells (American Type Culture Collection) were maintained in DMEM (Gibco) with $10 \%$ bovine growth serum (BGS; HyClone). Cells were transiently transfected with the MR expression plasmids and luciferase reporter plasmids (described below) by FuGene transfection reagent (Life Technologies).

miRNA Isolation and Microarray Hybridization. Total aortic miRNA was extracted from 3 or 12 month old mice using QIAzol (Qiagen), followed by column purification (miRNeasy; QIAGEN). Reversetranscribed aortic miRNA was hybridized to Mouse miRNA2.0 microarrays (Affymetrix) at the Boston University Medical School Center for Genetics and Genomics. Raw Affymetrix CEL files were normalized to produce gene-level expression values using the implementation of the Robust Multiarray Average (RMA) (58) in the affy package (59) included within in the Bioconductor software suite (version 2.10.0) (60). Affymetrix probeset mapping (also obtained from Bioconductor version 2.10.0) was used to normalize the miRNA 2.0 microarray. Differential miRNA expression was assessed with the limma package by creating linear models or using the empirical Bayes (moderated) $t$ test. Correction for multiple hypothesis testing was accomplished using the Benjamini-Hochberg false discovery rate (FDR) (61). All microarray analyses were performed using the R environment for statistical computing (version 2.12.0) (62). Complete vascular aging miR expression data are available at National Center for Biotechnology Information Gene Expression Omnibus (accession number: GSE84960).

Target filter analysis. Target filter analysis was performed using Ingenuity pathway analysis (Ingenuity Systems, www.ingenuity.com, IPA version: 8.8 (2010), Content version: 3204 (2010)) to identify potential target genes of individual miRNAs. The target filter analysis tool was used on miRNAs that were significantly altered with aging in MR-intact mice. Filters were applied for miRNA-mRNA pairings that were involved in cardiovascular signaling and either an experimentally observed or highly predicted miRNA-mRNA pairing.

Quantitative RT-PCR. Total RNA/miRNA was extracted from mouse vessels and cultured cells, reverse transcribed, and quantitative RT-PCR was performed with gene-specific primers as previously described (63). For mRNA studies, $C_{t}$ values were normalized to $\beta 2$-microglobulin $(B 2 m)$. For miRNA studies, $C t$ values were normalized to U6. Each PCR was performed in triplicate. Specific primers are specified in Supplemental Table 4.

Expression and reporter plasmids. The MR expression plasmid contains the full-length human MR cDNA [a gift from R. Evans, Gene Expression Laboratory, Salk Institute, La Jolla, CA (64)] cloned into the CMX expression vector with an N-terminal hemagluttanin (HA) tag. The MR reporter plasmid contains the mouse mammary tumor virus (MMTV) long-terminal repeat (65) cloned into the PGL2 luciferase reporter vector (Promega). The miR-155 host gene promoter [a gift from E. Flemington, Department of Pathology, Tulane University School of Medicine, New Orleans, LA (26)] contains the human miR-155 host gene promoter from -1494 to +228 relative to the transcription start site and was cloned into the basic PGL3 luciferase reporter vector.

Transfections and luciferase assay. Luciferase reporter assays were performed as described (66). Briefly, HEK293 cells were co-transfected with plasmids expressing the MR at increasing doses (1 microgram $(\mu \mathrm{g}), 2 \mu \mathrm{g}, 4 \mu \mathrm{g}$ ), a MMTV (MR response element)-luciferase reporter plasmid (65), or the miR-155 host gene promoter (26), and a $\beta$-galactosidase plasmid to normalize for transfection efficiency in $10 \%$ fetal bovine serum. Twenty-four hours later, cells were switched to serum free media and treated with vehicle or $10 \mathrm{~nm}$ aldosterone for 18 hours. Quantification of luciferase activity with a luciferase assay kit (Promega) was normalized to $\beta$-galactosidase activity assessed with Tropic accelerator (Applied Biosystems). Each treatment was carried out in triplicate and was performed in a minimum of three independent experiments.

Mouse smooth muscle cell harvest and culture. Mouse mesenteric and aortic smooth muscle cells were obtained using the explant procedure, as previously published (66). Briefly, mouse aortas or mesenteric arteries were isolated under sterile conditions and placed into a $100-\mathrm{mm}$ dish containing media. The adventitia was removed and the aorta was cut horizontally into 10 to 15 pieces. Each piece was placed into a 6- or 12-well collagen Biocoat plate (Falcon). Explants were cultured in low glucose, phenol red-free Dulbecco's modified Eagle's medium (DMEM) containing antibiotics and 10\% bovine growth serum (BGS) for 3 to 7 days. When the well was $50 \%$ to $75 \%$ confluent, the explants were removed, and the smooth muscle cells were cultured and passaged as needed. 
Mouse smooth muscle cell transfection. Smooth muscle cell transfections were performed with Lipofectamine RNAiMAX Transfection Reagent (Life Technologies), according to the manufacturer's protocol. Cells were transfected with the miR-155 mimic or the control scrambled miR (Life Technologies) for 48 hours, according to the supplier's instructions.

Blood pressure measurement by telemetry. Blood pressure studies were performed using implantable blood pressure transmitters (Data Sciences International, TA11PA-C10) with N=4-10 mice per group. Blood pressure was recorded for 60 seconds every $30 \mathrm{~min}$ as previously described (15). Mice were maintained on a 12 hour light-dark cycle, with normal chow $(0.3 \% \mathrm{NaCl}$; Harlan diet TD8604) and water available ad libitum.

Mesenteric vessel pressure myograph studies. Second and third order mesenteric resistance vessels were cannulated in a pressure myograph (Living Systems Instrumentation) and myogenic reactivity was measured over a range of intraluminal pressures from $10-120 \mathrm{mmHg}$ in $\mathrm{Ca}^{2+}$-containing PSS. After active tone measurements, vessels were superfused with buffer lacking added $\mathrm{Ca}^{2+}$ and containing $2 \mathrm{mM}$ EGTA. Passivediameter responses were then recorded over the pressure range $10-120 \mathrm{~mm} \mathrm{Hg}$. Tone was calculated as the percentage decrease in luminal diameter (LD) from the passive LD at $70 \mathrm{~mm} \mathrm{Hg:} \mathrm{\%} \mathrm{tone}=[1-$ (active diameter / passive diameter) $] \times 100$ as described (15).

Whole-cell $\mathrm{Ca}^{+2}$ channel recordings. Mesenteric artery SMCs were freshly dispersed as previously described $(15,66)$. Whole cell LTCC currents were recorded using a standard whole-cell patch clamp technique $(15,67)$. Cells were superfused with physiological saline solution (PSS) containing (in mM): 138 tetraethylammonium chloride (TEA-Cl), $0.1 \mathrm{CaCl} 2,1 \mathrm{MgCl} 2,5 \mathrm{KCl}, 10 \mathrm{HEPES}, 10$ Glucose, 20 barium chloride (BaCl2) pH 7.35 (Osm 300 Osm/L). Pipette solution (in mM):120 CsCl, 10 TEA-Cl, 10 EGTA, 1MgCl2, 15 HEPES, 5 Na2ATP, 0.5 Tris-GTP, $0.1 \mathrm{CaCl}$, pH 7.2. LTCC I-V curves were obtained by using a holding potential of -70 $\mathrm{mV}$, with step changes in potential from -60 to $+50 \mathrm{mV}$, duration of $400 \mathrm{~ms}$. Raw current values were normalized to cell capacitance and expressed as current density (pA pF-1).

Fura-2 Photometry Studies. Mesenteric arterioles were isolated, cannulated, pressurized to $70 \mathrm{mmHg}$ and loaded abluminally with $2 \mu \mathrm{M}$ fura-2 AM for 1 hour followed by 30 min washout. Fura- 2 ratios were determined after treatment with $100 \mathrm{~nm}$ BayK8644. Fura-2 data is presented as peak change from baseline of the $340 / 380$ ratio.

Mesenteric vessel wire myograph studies. Rings from second and third order mesenteric resistance arteries (MRAs) were mounted in a myograph (Danish Myo Technologies) for isometric tension recordings using PowerLab software (AD Instruments). A total of four rings per mouse were used, with $n=3-5$ mice for each wire myograph study. Rings were placed under a resting tension of $2 \mathrm{mN}$ in tissue baths containing warmed $\left(37^{\circ} \mathrm{C}\right)$, aerated $\left(95 \% \mathrm{O}_{2}, 5 \% \mathrm{CO}_{2}\right)$ standard physiological saline solution (PSS) (in $\mathrm{mM}$ : $130 \mathrm{NaCl}, 4.7 \mathrm{KCl}, 1.17 \mathrm{MgSO}_{4}, 0.03 \mathrm{EDTA}, 1.6 \mathrm{CaCl}_{2}, 14.9 \mathrm{NaHCO}_{3}, 1.18 \mathrm{KH}_{2} \mathrm{PO}_{4}$ and 5.5 glucose). Administration of $10 \mu \mathrm{M}$ phenylephrine ( $\mathrm{PE}$ ) was used to test arterial viability, and the presence of intact endothelium was verified by acetylcholine (Ach, $1 \mu \mathrm{M}$ )-induced relaxation of a half-maximal PE-induced contraction. Cumulative concentration-response curves for BayK8644 (10pM - $1 \mu \mathrm{M})$ and Angiotensin II (1-100nM) were constructed.

Lentiviral microRNA-155 Transduction. Recombinant mouse microRNA-155 lentivirus under the control of the mouse smooth muscle cell (SMC)-specific promoter SM22 (mSm22-mmu-mir-155) and mouse SM22 control lentivirus (mSm22-mmu-mir-ctrl) were purchased from Biosettia (Biosettia Inc). To generate the former lentivirus, mouse microRNA-155 was cloned within the intron of the human EF1 promoter region and the human EF1 promoter was replaced with the SMC-specific minimal mouse SM22 promoter $(68,69)$ in a self-inactivated lentiviral vector containing the red fluorescent puromycin-N-acetyl transferase gene. The resultant $\mathrm{pLV}$-microRNA vector was cotransfected with lentiviral packaging vector mix into HEK 293T cells to synthesize the final stock of microRNA-155 lentivirus that coexpresses the minimal mouse SM22 promoter.

Mice were injected via tail veins every other day for a total of 5 injections. For each injection, $0.2 \mathrm{~mL}$ of the concentrated viral suspension with a titer of $1 \times 10^{7} \mathrm{IU} / \mathrm{mL}$ was administered, as described by others (69). Mice were euthanized 10-14 days after the final injection and mesenteric vessels were harvested for wire myography and carotid vessels for DHE staining. To evaluate the efficacy and persistence of lentiviral transduction in our model, aortas were harvested simultaneously and subjected to MR, microRNA-155, and target gene expression analyses via standard quantitative polymerase chain reaction (PCR).

Dihydroethidium staining. Superoxide accumulation in carotid arteries was measured using dihydroethidium (DHE) staining. Right and left carotid arteries were isolated and exposed for $30 \mathrm{~min}$ to 
Krebs-HEPES buffer (in mM: $140 \mathrm{NaCl}, 5 \mathrm{KCl}, 1.6 \mathrm{CaCl}_{2}, 1 \mathrm{MgCl}_{2}, 8$ Hepes, 500 EDTA, and 11.1 glucose; pH 7.4) in the presence of vehicle or $200 \mathrm{nM}$ angiotensin II. Next, arteries were embedded in freezing medium (Tissue-Tek OCT compound) and transverse sections $(14 \mu \mathrm{m})$ were obtained with a cryostat $\left(-20^{\circ} \mathrm{C}\right)$. Carotid sections were incubated for $30 \mathrm{~min}$ at $37^{\circ} \mathrm{C}$ in a light-protected humidified chamber with Krebs-HEPES buffer containing the fluorescent dye-based dihydroethidium (DHE, $2 \mu \mathrm{M}$; Life Technology), used as ROS sensor. Images were obtained with a fluorescence microscope (Eclipse Ti, Nikon) using a $20 \times$ objective. The fluorescence was quantified by a blinded investigator by calculating the integrated density with respect to time using Image J software (National Institutes of Health).

Human serum collection and analyses. Blood was collected after a 12 hour overnight fast prior to randomization and after 1 month of eplerenone or placebo. Serum was isolated and frozen at $-80^{\circ} \mathrm{F}$. Serum miRNA was isolated via miRNA Serum/Plasma kit (Qiagen) according to the manufacturer's protocol. Serum miRNA was reverse transcribed using the miscript RT II kit (Qiagen) and expression was determined via qRT/PCR using human miR-155 primers (Qiagen), normalized to spike-in control C-elegans 39.

Statistics. Within-group differences were assessed with two-factor or three-factor analysis of variance (ANOVA) or repeated-measures ANOVA with Tukey or Bonferroni post hoc testing. Paired and unpaired two-tailed t-tests were performed where applicable. Pearson correlations were used for human serum analyses. $P<0.05$ was considered significant. Data are presented as mean \pm SEM.

Study approval. All mice were handled in accordance with US National Institutes of Health standards, and all procedures were approved by the Tufts Medical Center and University of Missouri Institutional Animal Care and Use Committees. Mice with inducible deletion of the mineralocorticoid receptor (MR) gene (Nr3c2) from smooth muscle cells (SMC-MR-KO mice) were generated by crossing floxed $\mathrm{MR}\left(\mathrm{MR}^{\mathrm{f} / \mathrm{f}}\right)$ mice with SMA-Cre-ER ${ }^{\mathrm{T} 2}$ mice (smooth muscle actin promoter driving expression of Cre-ER ${ }^{\mathrm{T} 2}$ recombinase that is activated by tamoxifen) as previously described $(15,70)$. For all studies, male $\mathrm{MR}^{\mathrm{f} / \mathrm{f}} / \mathrm{SMA}-\mathrm{Cre}-$ $\mathrm{ER}^{\mathrm{T} 2}$-negative (MR-intact) and $\mathrm{MR}^{\mathrm{f} / \mathrm{f}} / \mathrm{SMA}-\mathrm{Cre}-\mathrm{ER}^{\mathrm{T} 2}$-positive (SMC-MR-KO) littermates were treated with tamoxifen at 6-8 weeks of age resulting in SMC-specific MR deletion in the Cre positive animals as previously confirmed (15). For all studies, "young mice" are defined as 3-4 months of age and "aged mice" are defined as 12-15 months of age.

The human clinical study was carried out in accordance with the declaration of Helsinki and was approved by the Institutional Review Boards of the University of Florida, Texas A\&M University, Scott \& White Health System, and Tufts Medical Center. The purpose and risks were explained to each patient in full and written consent was obtained before beginning study participation. Healthy adults ages 59-77 (Characteristics, Supplemental Table 3) were enrolled in a randomized, double-blind placebo controlled cross-over study of the MR antagonist eplerenone (100 mg/day for 1 month) with 1-month washout between treatments as previously reported (23).

\section{Author contributions}

JJD, AM, APD, JM, SBB, KH, YY, JY, MA, WEB performed experiments. JJD, AM, APD, JM, SBB, KH, YY, DDC, MAH, IZJ analyzed data. JJD, SBB, DDC, MAH, IZJ designed experiments. JJD, MAH, IZJ performed statistical analyses. JJD, MAH, IZJ wrote the manuscript.

\section{Acknowledgments}

This work was supported by grants from the National Institutes of Health: HL095590 and HL119290 to IZJ and AG032067 to DDC, the American Heart Association: EIA18290005 to IZJ, 15POST21300000 to JJD and 08651174 to DDC, and the Department of Veterans Affairs BLR\&D: CDA-2 BX002030 to SBB. This work was also supported by resources and the use of facilities at the Harry S Truman Memorial Veterans Hospital in Columbia, MO. We acknowledge Adam Gower and the Boston University Microarray and Sequencing Resource Core Facility, as well as CTSA grant UL1-TR000157.

Address correspondence to: Iris Z. Jaffe, Tufts Medical Center, 800 Washington Street, Box 80.

Boston, Massachusetts 02111, USA. Phone: 617.636.0620; E-mail: ijaffe@tuftsmedicalcenter.org. 
1. Barton M, Husmann M, Meyer MR. Accelerated Vascular Aging as a Paradigm for Hypertensive Vascular Disease: Prevention and Therapy. Can J Cardiol. 2016;32(5):680-686.e4.

2. Gradman AH. Role of angiotensin II type 1 receptor antagonists in the treatment of hypertension in patients aged $>$ or $=65$ years. Drugs Aging. 2009;26(9):751-767.

3. Hyman DJ, Taffet GE. Blood pressure control in the elderly: can you have too much of a good thing?. Curr Hypertens Rep. 2009;11(5):337-342

4. Ungvari Z, Kaley G, de Cabo R, Sonntag WE, Csiszar A. Mechanisms of vascular aging: new perspectives. J Gerontol A Biol Sci Med Sci. 2010;65(10):1028-1041.

5. Pitt B, et al. The effect of spironolactone on morbidity and mortality in patients with severe heart failure. Randomized Aldactone Evaluation Study Investigators. N Engl J Med. 1999;341(10):709-717.

6. Armstrong C, Joint National Committee. JNC8 guidelines for the management of hypertension in adults. Am Fam Physician 2014;90(7):503-504.

7. SPRINT Research Group, et al. A Randomized Trial of Intensive versus Standard Blood-Pressure Control. N Engl J Med. 2015;373(22):2103-2116.

8. Williamson JD, et al. Intensive vs Standard Blood Pressure Control and Cardiovascular Disease Outcomes in Adults Aged $\geq 75$ Years: A Randomized Clinical Trial. JAMA. 2016;315(24):2673-2682.

9. Bitzer M, Wiggins J. Aging Biology in the Kidney. Adv Chronic Kidney Dis. 2016;23(1):12-18.

10. Joseph BK, Thakali KM, Moore CL, Rhee SW. Ion channel remodeling in vascular smooth muscle during hypertension: Implications for novel therapeutic approaches. Pharmacol Res. 2013;70(1):126-138.

11. Wang M, Monticone RE, Lakatta EG. Arterial aging: a journey into subclinical arterial disease. Curr Opin Nephrol Hypertens. 2010;19(2):201-207.

12. Jaffe IZ, Tintut Y, Newfell BG, Demer LL, Mendelsohn ME. Mineralocorticoid receptor activation promotes vascular cell calcification. Arterioscler Thromb Vasc Biol. 2007;27(4):799-805

13. Jaffe IZ, et al. Placental growth factor mediates aldosterone-dependent vascular injury in mice. J Clin Invest. 2010;120(11):3891-3900

14. Jaffe IZ, Mendelsohn ME. Angiotensin II and aldosterone regulate gene transcription via functional mineralocortocoid receptors in human coronary artery smooth muscle cells. Circ Res. 2005;96(6):643-650.

15. McCurley A, et al. Direct regulation of blood pressure by smooth muscle cell mineralocorticoid receptors. Nat Med. 2012;18(9):1429-1433.

16. Dimmeler S, Nicotera P. MicroRNAs in age-related diseases. EMBO Mol Med. 2013;5(2):180-190.

17. Noren Hooten N, Abdelmohsen K, Gorospe M, Ejiogu N, Zonderman AB, Evans MK. microRNA expression patterns reveal differential expression of target genes with age. PLoS One. 2010;5(5):e10724.

18. Krug AW, et al. Elevated mineralocorticoid receptor activity in Aged Rat vascular smooth muscle cells Promotes a Proinflammatory Phenotype Via ERK1/2 MAPK and EGFR-Dependent Pathways. Hypertension. 2010;55(6):1476-1483

19. Chen NX, et al. Decreased microRNA is involved in the vascular remodeling abnormalities in chronic kidney disease (CKD). PLoS One. 2013;8(5):e64558.

20. Zheng L, et al. MicroRNA-155 regulates angiotensin II type 1 receptor expression and phenotypic differentiation in vascular adventitial fibroblasts. Biochem Biophys Res Commun. 2010;400(4):483-488.

21. Poduri A, et al. Regional variation in aortic AT1b receptor mRNA abundance is associated with contractility but unrelated to atherosclerosis and aortic aneurysms. PLoS One. 2012;7(10):e48462.

22. Hill MA, Davis MJ, Meininger GA, Potocnik SJ, Murphy TV. Arteriolar myogenic signalling mechanisms: Implications for local vascular function. Clin Hemorheol Microcirc. 2006;34(1-2):67-79.

23. Hwang MH, et al. Mineralocorticoid receptors modulate vascular endothelial function in human obesity. Clin Sci. 2013;125(11):513-520.

24. Lee S, Choi E, Cha MJ, Park AJ, Yoon C, Hwang KC. Impact of miRNAs on cardiovascular aging. J Geriatr Cardiol. 2015;12(5):569-574

25. Bretschneider M, et al. Activated mineralocorticoid receptor regulates micro-RNA-29b in vascular smooth muscle cells. FASEB J. 2016;30(4):1610-1622.

26. Yin Q, Wang X, McBride J, Fewell C, Flemington E. B-cell receptor activation induces BIC/miR-155 expression through a conserved AP-1 element. J Biol Chem. 2008;283(5):2654-2662.

27. Onyeagucha BC, Mercado-Pimentel ME, Hutchison J, Flemington EK, Nelson MA. S100P/RAGE signaling regulates microRNA-155 expression via AP-1 activation in colon cancer. Exp Cell Res. 2013;319(13):2081-2090.

28. Bender SB, McGraw AP, Jaffe IZ, Sowers JR. Mineralocorticoid receptor-mediated vascular insulin resistance: an early contributor to diabetes-related vascular disease?. Diabetes. 2013;62(2):313-319.

29. Bender SB, et al. Mineralocorticoid receptor antagonism treats obesity-associated cardiac diastolic dysfunction. Hypertension. 2015;65(5):1082-1088

30. Ratman D, et al. How glucocorticoid receptors modulate the activity of other transcription factors: a scope beyond tethering Mol Cell Endocrinol. 2013;380(1-2):41-54.

31. Chinenov Y, Gupte R, Rogatsky I. Nuclear receptors in inflammation control: repression by GR and beyond. Mol Cell Endocrinol. 2013;380(1-2):55-64.

32. Biddie SC, et al. Transcription factor AP1 potentiates chromatin accessibility and glucocorticoid receptor binding. $\mathrm{Mol}$ Cell. 2011;43(1):145-155.

33. Nagase M, Fujita T. Role of Rac1-mineralocorticoid-receptor signalling in renal and cardiac disease. Nat Rev Nephrol. 2013;9(2):86-98.

34. Ceolotto G, et al. Interplay between miR-155, AT1R A1166C polymorphism, and AT1R expression in young untreated hypertensives. Am J Hypertens. 2011;24(2):241-246

35. Pratt PF, Bonnet S, Ludwig LM, Bonnet P, Rusch NJ. Upregulation of L-type Ca2+ channels in mesenteric and skeletal arteries of SHR. Hypertension. 2002;40(2):214-219.

36. Kazda S, Garthoff B, Luckhaus G. Calcium and malignant hypertension in animal experiment: effects of experimental manipu- 
lation of calcium influx. Am J Nephrol. 1986;6 Suppl 1:145-150.

37. Suzuki H, Zweifach BW, Schmid-Schönbein GW. Glucocorticoid modulates vasodilator response of mesenteric arterioles in spontaneously hypertensive rats. Hypertension. 1996;27(1):114-118.

38. Falcone JC, Granger HJ, Meininger GA. Enhanced myogenic activation in skeletal muscle arterioles from spontaneously hypertensive rats. Am J Physiol. 1993;265(6 Pt 2):H1847-H1855.

39. Arii T, Ohyanagi M, Shibuya J, Iwasaki T. Increased function of the voltage-dependent calcium channels, without increase of $\mathrm{Ca} 2+$ release from the sarcoplasmic reticulum in the arterioles of spontaneous hypertensive rats. Am J Hypertens. 1999;12(12 Pt 1-2):1236-1242.

40. Ohya Y, Abe I, Fujii K, Takata Y, Fujishima M. Voltage-dependent Ca2+ channels in resistance arteries from spontaneously hypertensive rats. Circ Res. 1993;73(6):1090-1099.

41. Cox RH, Lozinskaya IM. Augmented calcium currents in mesenteric artery branches of the spontaneously hypertensive rat. Hypertension. 1995;26(6 Pt 2):1060-1064.

42. Lozinskaya IM, Cox RH. Effects of age on Ca2+ currents in small mesenteric artery myocytes from Wistar-Kyoto and spontaneously hypertensive rats. Hypertension. 1997;29(6):1329-1336.

43. Ohya Y, Tsuchihashi T, Kagiyama S, Abe I, Fujishima M. Single L-type calcium channels in smooth muscle cells from resistance arteries of spontaneously hypertensive rats. Hypertension. 1998;31(5):1125-1129.

44. Sun HX, et al. Essential role of microRNA-155 in regulating endothelium-dependent vasorelaxation by targeting endothelial nitric oxide synthase. Hypertension. 2012;60(6):1407-1414.

45. Vigorito E, Kohlhaas S, Lu D, Leyland R. miR-155: an ancient regulator of the immune system. Immunol Rev. 2013;253(1):146-157.

46. Caballero-Garrido E, et al. In Vivo Inhibition of miR-155 Promotes Recovery after Experimental Mouse Stroke. J Neurosci. 2015;35(36):12446-12464.

47. Bobo D, Robinson KJ, Islam J, Thurecht KJ, Corrie SR. Nanoparticle-based medicines: A review of FDA-approved materials and clinical trials to date [published online ahead of print June 14, 2016]. Pharm Res. doi:10.1007/s11095.016.1958-5.

48. Kwon EJ, Skalak M, Lo Bu R, Bhatia SN. A neuron-targeted nanoparticle for siRNA delivery to traumatic brain injuries [published online ahead of print July 18, 2016]. ACS Nano. doi: 10.1021/acsnano.6b03858.

49. Swain S, Babu SM, Beg S, Jena J. Nanoparticles for cancer targeting: Current and Future Directions [published online ahead of print July 13, 2016]. Curr Drug Deliv.

50. Sundaramoorthy P, Ramasamy T, Mishra SK, Jeong KY, Yong CS, Kim JO, Kim HM. Engineering of caveolae-specific selfmicellizing anticancer lipid nanoparticles to enhance the chemotherapeutic efficacy of oxaliplatin in colorectal cancer cells [published online ahead of print July 6, 2016]. Acta Biomater. doi: 10.1016/j.actbio.2016.07.006.

51. Iqbal J, Parviz Y, Pitt B, Newell-Price J, Al-Mohammad A, Zannad F. Selection of a mineralocorticoid receptor antagonist for patients with hypertension or heart failure. Eur J Heart Fail. 2014;16(2):143-150.

52. Calhoun DA. Low-dose aldosterone blockade as a new treatment paradigm for controlling resistant hypertension. J Clin Hypertens (Greenwich). 2007;9(1 Suppl 1):19-24.

53. Herrera M, Sparks MA, Alfonso-Pecchio AR, Harrison-Bernard LM, Coffman TM. Lack of specificity of commercial antibodies leads to misidentification of angiotensin type 1 receptor protein. Hypertension. 2013;61(1):253-258.

54. dos Santos RL, da Silva FB, Ribeiro RF, Stefanon I. Sex hormones in the cardiovascular system. Horm Mol Biol Clin Investig. 2014;18(2):89-103.

55. Hay M. Sex, the brain and hypertension: brain oestrogen receptors and high blood pressure risk factors. Clin Sci. 2016;130(1):9-18.

56. Glicklich D, Frishman WH. Drug therapy of apparent treatment-resistant hypertension: focus on mineralocorticoid receptor antagonists. Drugs. 2015;75(5):473-485.

57. Clark D, Ahmed MI, Calhoun DA. Resistant hypertension and aldosterone: an update. Can J Cardiol. 2012;28(3):318-325.

58. Irizarry RA, et al. Exploration, normalization, and summaries of high density oligonucleotide array probe level data. Biostatistics. 2003;4(2):249-264.

59. Gautier L, Cope L, Bolstad BM, Irizarry RA. affy--analysis of Affymetrix GeneChip data at the probe level. Bioinformatics. 2004;20(3):307-315.

60. Gentleman RC, et al. Bioconductor: open software development for computational biology and bioinformatics. Genome Biol. 2004;5(10):R80.

61. Benjamini Y, Hochberg Y. Controlling the false discovery rate: a practical and powerful approach to multiple testing. JR Stat Soc Series B Stat Methodol. 1995:57(1):289-300.

62. R Development Core Team. The R Project for Statistical Computing. http://www.R-project.org. Accessed: August 3 , 2016.

63. Newfell BG, et al. Aldosterone regulates vascular gene transcription via oxidative stress-dependent and -independent pathways. Arterioscler Thromb Vasc Biol. 2011;31(8):1871-1880.

64. Arriza JL, et al. Cloning of human mineralocorticoid receptor complementary DNA: structural and functional kinship with the glucocorticoid receptor. Science. 1987;237(4812):268-275.

65. Lombès M, Binart N, Oblin ME, Joulin V, Baulieu EE. Characterization of the interaction of the human mineralocorticosteroid receptor with hormone response elements. Biochem J. 1993;292(Pt 2):577-583.

66. Barrett Mueller K, et al. Estrogen receptor inhibits mineralocorticoid receptor transcriptional regulatory function. Endocrinology. 2014;155(11):4461-4472.

67. Yang Y, et al. Heterogeneity in function of small artery smooth muscle BKCa: involvement of the beta1-subunit. JPhysiol (Lond). 2009;587(Pt 12):3025-3044.

68. Moessler H, Mericskay M, Li Z, Nagl S, Paulin D, Small JV. The SM 22 promoter directs tissue-specific expression in arterial but not in venous or visceral smooth muscle cells in transgenic mice. Development. 1996;122(8):2415-2425.

69. Lovren F, et al. MicroRNA-145 targeted therapy reduces atherosclerosis. Circulation. 2012;126(11 Suppl 1):S81-S90

70. Wendling O, Bornert JM, Chambon P, Metzger D. Efficient temporally-controlled targeted mutagenesis in smooth muscle cells of the adult mouse. Genesis. 2009;47(1):14-18. 